\title{
On Cylindrical Graph Construction and its Applications
}

\author{
Amir Daneshgar \\ Department of Mathematical Sciences \\ Sharif University of Technology \\ Tehran, Iran. \\ daneshgar@sharif .ir
}

\author{
Mohsen Hejrati* \\ Department of Computer Science \\ University of California, Irvine \\ USA \\ shejrati@ics.uci.edu
}

\author{
Meysam Madani \\ Department of Mathematical Sciences \\ Sharif University of Technology \\ Tehran, Iran. \\ madani@mehr.sharif.ir
}

Submitted: Aug 15, 2014; Accepted: Feb 2, 2016; Published: Feb 19, 2016

Mathematics Subject Classifications: 05C15, 05C60, 05C76.

\begin{abstract}
In this article we introduce the cylindrical construction, as an edge-replacement procedure admitting twists on both ends of the hyperedges, generalizing the concepts of lifts and Pultr templates at the same time. We prove a tensor-hom duality for this construction and we show that not only a large number of well-known graph constructions are cylindrical but also the construction and its dual give rise to some new graph constructions, applications and results. To show the applicability of the main duality we introduce generalized Grötzsch, generalized Petersen-like and Coxeter-like graphs and we prove some coloring properties of these graphs.
\end{abstract}

Keywords: graph homomorphism, cylindrical construction, generalized Petersen graph, generalized Mycielski construction, Coxeter-like graphs, adjoint functor, category of graphs, labeled and marked graphs, extremal problems.

\section{Motivations and background}

This article is about a tensor-hom duality for a specific graph construction that will be called the cylindrical construction and can be described as replacement of edges of a graph

\footnotetext{
*M. Hejrati's contributions to this article were obtained when he was a BSc. student at Sharif University of Technology.
} 
by some other graph(s), here called cylinders $^{1}$, admitting twists at each end. Not only the construction essentially generalizes most well-known operations on graphs but also it will give rise to a number of new constructions, concepts and methods. In Section 5 we presents a couple of examples to show how the new approach can be used, while the results of this section can also be of independent interest.

To begin, it is instructive to mention how the construction can be described as a generalization of lifts and Pultr functors at the same time (also see the last paragraph of Section 3.1).

For the case of 2-lifts, consider different 2-lifts of a triangle (i.e. the complete graph $\mathrm{K}_{3}$ ) that intuitively can be described as a process of replacing edges of the triangle by matchings of size 2, while one is allowed to arbitrarily twist each one of the ends of each matching. Figure 1 illustrates two of such 2-lifts showing that if there is no twist then one ends up with a pair of triangles while if one uses only one twisted matching then the outcome will be the 6 -cycle $\mathrm{C}_{6}$. Note that this very simple example clearly shows the effect of twists on the connectivity of the construction. The breakthrough of A. W. Marcus, D. A. Spielman and N. Srivastava [18] proves that this phenomenon is typical (at least for bipartite graphs) in the sense that there are highly connected random 2-lifts of a highly connected graph (for details and more on 2-lifts see $[1,18]$ ).
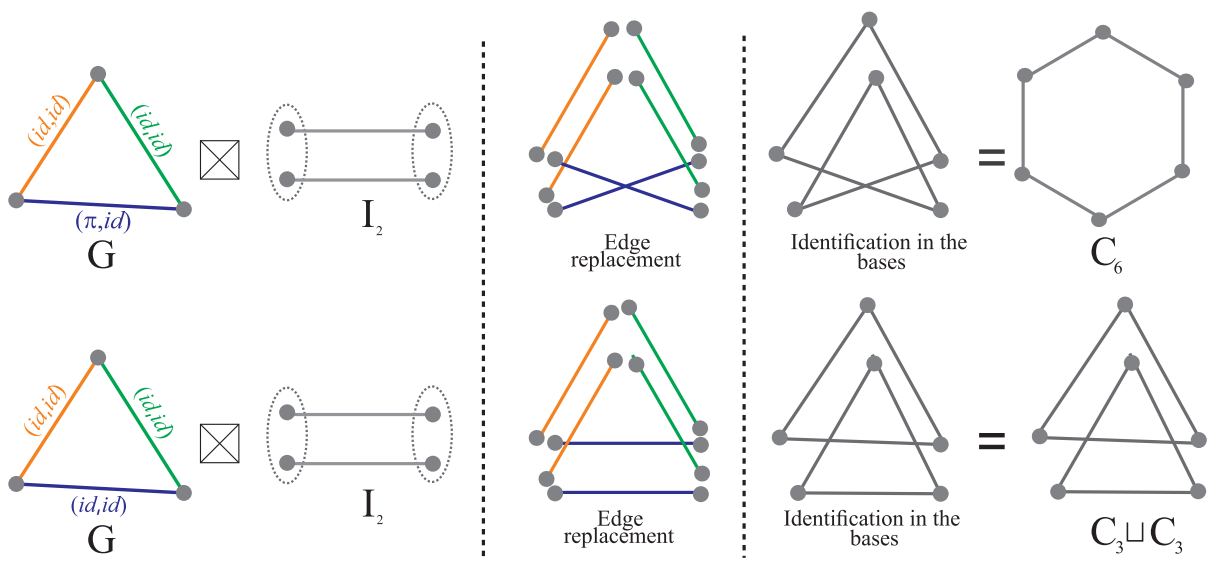

Figure 1: The role of twists in 2-lifts.

On the other hand, one may consider the Pultr universal adjunction construction [23] that for the case of directed graphs can be described as follows (also see [10]).

A Pultr template is a quadruple $\tau=\left(\mathrm{B}, \mathrm{C}, \eta_{1}, \eta_{2}\right)$, where $\mathrm{B}$ and $\mathrm{C}$ are digraphs and $\eta_{1}, \eta_{2}: \mathrm{B} \rightarrow \mathrm{C}$ are digraph homomorphisms. For the time being, just for the sake of intuition, let us consider $\mathrm{C}$ as a cylinder with $\eta_{1}(\mathrm{~B})$ and $\eta_{2}(\mathrm{~B})$ as bases (see Figure 2). In this setting, Pultr's left adjoint functor $-\otimes \tau$ acting on a digraph $\mathrm{G}$ can be described as a digraph $\mathrm{G} \otimes \tau$ that can be constructed by taking a copy of the template $\tau_{e}$ for each

\footnotetext{
${ }^{1}$ The concept of a cylinder may be compared to that of a hyperedge as it is used in the literature of graph grammars (e.g. see [13]) and must not be confused with the same word used in the theory of hypergraphs.
} 
edge $e$ of $\mathrm{G}$ and then identifying the corresponding bases for each vertex of $\mathrm{G}$. A result of Pultr [23] implies that this admits a right adjoint functor (between thin categories) as $[\tau,-]$ where acting on a digraph $\mathrm{H}$ can be described as a digraph whose set of vertices consists of all digraph homomorphisms $\xi: \mathrm{B} \longrightarrow \mathrm{H}$ and there is a directed edge from $\xi_{1}$ to $\xi_{2}$ if there exists a homomorphism $\rho: \mathrm{C} \longrightarrow \mathrm{H}$ such that $\xi_{i}=\rho \circ \eta_{i}(i \in\{1,2\})$. It should be noted that the full power of the construction is preserved even if one considers templates for which $\eta_{1}$ and $\eta_{2}$ are isomorphisms on their ranges (i.e when they are oneto-one embeddings and $\eta_{1}(\mathrm{~B}) \simeq \eta_{2}(\mathrm{~B}) \simeq \mathrm{B}$ ) which justifies the intuitive consideration of templates as cylinders (e.g. see the remarks proceeding Definition 3). On the other hand, it must be noted that the intersection of ranges of $\eta_{1}$ and $\eta_{2}$ as bases may not be empty. This, in our opinion, is one of the interesting aspects of this construction that has not been emphasized appropriately and will be discussed in more detail in what follows (e.g. see $[4,8]$ for some applications and also Sections 3 and 5).

From this point of view, what is introduced in this article as the cylindrical construction can be described as a Pultr left adjoint construction using a set of templates (not necessarily of size one) in which one may also apply twists from the group of automorphisms of each base. The main result of this article is the fact that even in this general setting a similar Pultr adjunction holds.

Although, most of well-known graph operations can be interpreted as cylindrical constructions (see Section 3 and [7] for more examples), let us concentrate on some distinctive aspects of this construction in relation to some open questions.

To start, let us consider the graph homomorphism problem as one of the most fundamental problems in graph theory. From this point of view, strictly speaking, our main result states that the homomorphism problem $\mathrm{G} \otimes \mathrm{C} \stackrel{?}{\longrightarrow} \mathrm{H}$ for the cylindrical construct $\mathrm{G} \otimes \mathrm{C}$ is equivalent to the homomorphism problem $\mathrm{G} \stackrel{?}{\longrightarrow}[\mathrm{C}, \mathrm{H}]$ in which $[\mathrm{C}, \mathrm{H}]$ stands for the exponential (i.e. the right adjoint) construction. In particular, it is interesting to note that the first homomorphism problem is in the category of labeled graphs where the second homomorphism problem is in a specific category of labeled graphs (called $(\Gamma, m)$ graphs in the sequel) whose labels encode the information of twists and templates used in the cylindrical construction $\mathrm{G} \otimes \mathrm{C}$. This reduction can be used effectively if one can develop no-homomorphism theorems in the category of $(\Gamma, m)$-graphs, while one of our main objectives in Section 5 is to illustrate different aspects of this scenario using some specific examples. Although, these examples generalize some previously known results to

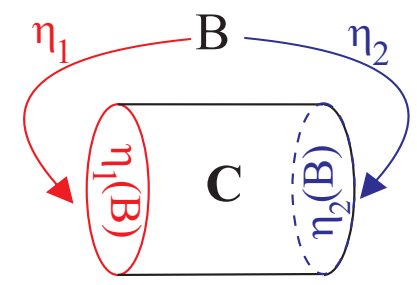

Figure 2: A Pultr template when the maps are embeddings and bases do not intersect. 
a broader class of graphs and may be of independent interest, the mentioned techniques opens a new approach to get no-homomorphism results through the label structure of $(\Gamma, m)$-graphs (also see $[20]$ ).

Nowadays, extremal sparse graphs is a classic subject in graph theory that contains many deep open questions. As mentioned by J. Nešetřil in [21],

"Many classical problems in this area still have no satisfactory answer. Particularly, the basic challenge in this area of complex large girth graphs is to find new constructions. The old questions remain. The recent advances of theoretical computer science put these problems in a new context and make these questions very actual."

Among important extremal conditions in this area one may refer to the smallest order, the largest spectral gap, the largest possible (ordinary) chromatic number or the largest circular chromatic number of sparse graphs, all of which somehow referring to maximal connectivity in some sense. Specifically, one may refer to the pentagon problem [22], stating whether there exists a threshold $g$ such that any 3-regular graph of girth larger than $g$ admits a homomorphism to the 5 -cycle $\mathrm{C}_{5}$. On the other hand, one may refer to the largest possible chromatic number of a $d$-regular graph of arbitrary large girth. In this regard, a result of A. V. Kostochka [2] states that this maximum chromatic number is less than or equal to $\frac{d}{2}+2$ (also see [20] for connections to Reed's conjecture). In a constructive approach to these problems finding constructions that do not reduce the girth and the parameter related to the extremal condition at the same time is of great importance. Again, examples discussed in Section 5 have been chosen to show the importance of twists in cylindrical constructions maintaining such properties. Generalizations of such constructions using tree-based cylinders that can be used to generate highly connected graphs of large girth and relate such extremal questions will appear elsewhere (also see $[20])$.

From another point of view, constructing highly connected sparse graphs, fits into the setup of random matrix theory. This angle is clearer when one concentrates on cylindrical constructions using random cylinders or twists. Note that our basic example of 2-lifts clearly illustrates the importance of this approach, while using cylindrical construction with properly chosen cylinders (as the $\sqcap$-cylinder introduced in Section 2) can be used to not only preserve connectivity and sparseness but also to go through a degree reduction process. In particular, we will show in the sequel that the Petersen graph is a $\sqcap$-lift of $\mathrm{K}_{5}$ which is the most connected graph on 5 vertices. Our examples and proposed techniques in Section 5 also show how one may use the results of this article to study the properties of such lifts. Specifically, following this line of thought, this gives rise to the question of whether iterated random $\sqcap$-lifts of $\mathrm{K}_{2^{t}+3}$ contain 3-regular graphs which are not $\mathrm{C}_{5}{ }^{-}$ colorable for each integer $t \geqslant 1$. On the other hand, considering the spectral gap, this may be described as a study of random structured matrices which is one of the most active and central subjects in modern physics and mathematics.

In Section 2 we go through the basic definitions and notations we will be needing hereafter. An important part of this framework is going through the definitions of different 
categories of graphs and the pushout construction in them.

In Secion 3 we introduce the main construction and its dual, in which we go through the details of expressing some different well-known and new graph constructions as a cylindrical product or its dual. In this section we have tried to avoid being formal ${ }^{2}$ and have described the constructions in detail from the scratch using a variant of examples for clarification.

In Section 4 we state and prove the main duality result and we present a categorical perspective showing the naturality of definitions.

Section 5 is dedicated to analysis of some selected examples. On the one hand, the analysis will give rise to new results clarifying and generalizing some known facts. On the other hand, the examples are carefully chosen to illustrate applications of our main results and methods.

To this end, first, we describe Mycielski's construction and its generalization as a cylindrical construction. In this regard we also consider a relaxation of this construction as a cylindrical construct using a class of two $(\Gamma, 2)$-coherent set of cylinders, and we analyze its properties using the fundamental duality theorem.

For the second example, we focus on generalized Petersen graphs for which we prove a positive as well as a negative homomorphism results for homomorphisms to the five-cycle. In particular, we show how nohomomorphism theorems at the level of $(\Gamma, m)$-graphs can be effectively used to prove nohomomorphism results for cylindrical constructs.

For the third example, we introduce Coxeter-like graphs. This case is studied to show that even using forgetful functors at the level of $(\Gamma, m)$-graphs may still give rise to nontrivial results, which can be considered as an evidence to the fact that the exponential construction as the dual to the cylindrical construction encodes a lot of information in its own structure regardless of its labels.

More details and some concluding results will appear in Section 6.

\section{Basic definitions}

In this section we go through some basic definitions and will set the notations for what will appear in the rest of the article. Throughout the paper, sets are denoted by capital Roman characters as $X, Y, \ldots$ while graphs are denoted by capital RightRoman ones as $\mathrm{G}$ or $\mathrm{H}$. Ordered lists are denoted as $\left(x_{1}, x_{2}, \ldots, x_{t}\right)$, and in a concise form by Bold small characters as $\mathbf{x}$. The notation $|$.$| is used for the size of a set as in |X|$ or for the size of a list as in $|\mathbf{x}|$.

We use $\widehat{1 \ldots k}$ to refer to the set $\{1,2, \ldots, k\}$, and the symbol $\mathbf{S}_{\mathbf{k}}$ is used to refer to the group of all $k$-permutations of this set. The symbol $i d$ stands for the identity of the group $\mathbf{S}_{\mathbf{k}}$, and the symbol $\left\lfloor a_{1}, a_{2}, \ldots, a_{t}\right\rceil$ stands for the cycle $\left(a_{1}, a_{2}, \ldots, a_{t}\right)$ in this group. Also, $\mathrm{id} \simeq \mathrm{S}_{\mathbf{1}} \leqslant \mathrm{S}_{\mathrm{k}}$ is the trivial subgroup containing only the identity element.

\footnotetext{
${ }^{2}$ Note that from a categorical point of view the natural framework to describe such a construction is the category of cospans, however, we have focused on the current literature and nomenclature of graph theory to avoid unnecessary complexities.
} 
For the ordered list $\mathbf{x}=\left(x_{1}, x_{2}, \ldots, x_{k}\right)$ and $\gamma \in \mathbf{S}_{\mathbf{k}}$, the right action of $\gamma$ on indices of $\mathbf{x}$ is denoted as $\mathbf{x} \gamma \stackrel{\text { def }}{=}\left(x_{\gamma(1)}, x_{\gamma(2)}, \ldots, x_{\gamma(k)}\right)$, where for any function $f$,

$$
f \mathbf{x} \stackrel{\text { def }}{=}\left(f\left(x_{1}\right), f\left(x_{2}\right), \ldots, f\left(x_{k}\right)\right) .
$$

We use the notation $\{\mathbf{x}\}$ for the set $\left\{x_{1}, x_{2}, \ldots, x_{k}\right\}$.

\subsection{Graphs and their categories}

In this paper, a graph $\mathrm{G}=\left(V(\mathrm{G}), E(\mathrm{G}), \iota_{\mathrm{G}}: E(\mathrm{G}) \longrightarrow V(\mathrm{G}), \tau_{\mathrm{G}}: E(\mathrm{G}) \longrightarrow V(\mathrm{G})\right)$ consists of a finite set of vertices $V(\mathrm{G})$, a finite set of edges $E(\mathrm{G})$ and two maps $\iota_{\mathrm{G}}$ and $\tau_{\mathrm{G}}$ called initial and terminal maps, respectively. Hence, our graphs are finite but are generally directed, and may contain loops or multiple edges. For any edge $e \in E(\mathrm{G})$, the vertex $u \stackrel{\text { def }}{=} \iota_{\mathrm{G}}(e)$ is called the initial vertex of $e$ and may be denoted by $e^{-}$. Similarly, the vertex $v \stackrel{\text { def }}{=} \tau_{\mathrm{G}}(e)$ is called the terminal vertex of $e$ and may be denoted by $e^{+}$. Two edges $e_{1}$ and $e_{2}$ are said to be similar if

$$
e_{1}^{-}=e_{2}^{-} \text {and } e_{1}^{+}=e_{2}^{+},
$$

and have the same label if the graph is labeled (see Definition 2). An edge $e$ is called a loop if $e^{-}=e^{+}$. A non-loop edge is an edge which is not a loop.

Given a graph $\mathrm{G}$ and a subset $X \subseteq V(\mathrm{G})$, the (vertex) induced subgraph on the subset $X$ is denoted by $\mathrm{G}[X]$. The vertex reduced part of a graph $\mathrm{G}$, denoted by $\operatorname{vred}(\mathrm{G})$, is defined to be $K_{1}$ when $G$ is an empty graph (i.e. when $G$ contains no edge) and is defined to be the subgraph (up to isomorphism) obtained from $G$ by excluding all isolated vertices (of degree zero) otherwise. Similarly, the edge reduced part of a graph G, denoted by ered $(\mathrm{G})$ is the subgraph (up to isomorphism) obtained from $\mathrm{G}$ by identifying similar multiple non-loop edges and identifying all but two of similar loops (i.e. when the number of similar loops is larger than two). The reduced part of a graph $\mathrm{G}$, denoted by $\operatorname{red}(G)$ is defined to be the subgraph ered $(\operatorname{vred}(G))$. A graph is said to be reduced if it is isomorphic to its reduced part, i.e. $\mathrm{G} \simeq \operatorname{red}(\mathrm{G})$.

On the other hand, a graph $\mathrm{G}$ is said to be symmetric if its edges can be partitioned into dual pairs as $e$ and $\tilde{e}$ such that $e^{-}=\tilde{e}^{+}$and $e^{+}=\tilde{e}^{-}$.

Note that the data contained in a symmetric graph can be encoded in a new undirected graph on the same vertex set where each dual pair is replaced by an undirected (i.e. simple) edge. An undirected graph without any loop or multiple edges is said to be a simple graph.

In this setting, when there is no ambiguity, the edge $e$ is sometimes referred to by the notation $u v$. Also, we say that an edge $e$ intersects a vertex $u$ if $u=e^{-}$or $u=e^{+}$. Hereafter, we may freely refer to an edge $u v$ when the corresponding data is clear from the context.

A (graph) homomorphism $\left(\sigma_{V}, \sigma_{E}\right)$ from a graph $\mathrm{G}=\left(V(\mathrm{G}), E(\mathrm{G}), \iota_{\mathrm{G}}, \tau_{\mathrm{G}}\right)$ to a graph $\mathrm{H}=\left(V(\mathrm{H}), E(\mathrm{H}), \iota_{\mathrm{H}}, \tau_{\mathrm{H}}\right)$ is a pair of maps

$$
\sigma_{V}: V(\mathrm{G}) \longrightarrow V(\mathrm{H}) \quad \text { and } \quad \sigma_{E}: E(\mathrm{G}) \longrightarrow E(\mathrm{H})
$$


which are compatible with the structure maps, i.e.,

$$
\iota_{\mathrm{H}} \circ \sigma_{E}=\sigma_{V} \circ \iota_{\mathrm{G}} \text { and } \tau_{\mathrm{H}} \circ \sigma_{E}=\sigma_{V} \circ \tau_{\mathrm{G}} .
$$

When there is no ambiguity we may exclude the subscript and use the notation $\sigma$ for both the vertex and edge maps when the mapping is clear from the operand. Also, note that when there is no multiple edge (e.g. for reduced undirected or simple graphs), one may think of $E(\mathrm{G})$ as a subset of $V(\mathrm{G}) \times V(\mathrm{G})$ and one may talk about the homomorphism $\sigma_{V}$ when the compatibility condition is equivalent to the following,

$$
u v \in E(\mathrm{G}) \quad \Rightarrow \quad \sigma(u) \sigma(v) \in E(\mathrm{H}) .
$$

Similar concepts can be defined for undirected graphs by forgetting directions.

The set of homomorphisms from the graph $G$ to the graph $H$ is denoted by $\operatorname{Hom}(G, H)$, and Grph stands for the category of (directed) graphs and their homomorphisms. The partial ordering $G \leqslant H$ on the set of all graphs is equivalent to the condition $\operatorname{Hom}(G, H) \neq$ $\emptyset$. Two graphs $\mathrm{G}$ and $\mathrm{H}$ are said to be homomorphically equivalent, denoted by $\mathrm{G} \approx \mathrm{H}$, if both sets $\operatorname{Hom}(\mathrm{G}, \mathrm{H})$ and $\operatorname{Hom}(\mathrm{H}, \mathrm{G})$ are non-empty i.e., if $\mathrm{G} \leqslant \mathrm{H}$ and $\mathrm{H} \leqslant \mathrm{G}$. Note that $\mathrm{G} \approx \operatorname{red}(\mathrm{G})$ holds for any graph $\mathrm{G}$.

For any category of graphs as $\mathbf{C}$, the corresponding thin category, denoted by $\mathbf{C}^{\leqslant}$, is the partially ordered set (as a category) with the same objects for which the hom-set $\mathrm{Hom}^{\leqslant}(\mathrm{G}, \mathrm{H})$ is nonempty (and in that case contains a fixed unique homomorphism) if and only if $\operatorname{Hom}(\mathrm{G}, \mathrm{H})$ is nonempty in C. For instance, Grph ${ }^{\leqslant}$stands for the thin category of (directed) graphs and their homomorphisms.

The interested reader is encouraged to refer to [15] for more on graphs and their homomorphisms.

\section{Definition 1. Marked graphs}

Let $X=\left\{x_{1}, x_{2}, \ldots, x_{k}\right\}$ and $\mathrm{G}$ be a set and a graph, respectively, and also, consider a one-to-one map $\varrho: X \hookrightarrow V(\mathrm{G})$. Evidently, one can consider $\varrho$ as a graph monomorphism from the empty graph $\mathrm{X}$ on the vertex set $X$ to the graph $\mathrm{G}$, where in this setting we interpret the situation as marking some vertices of $\mathrm{G}$ by the elements of $X$. The data introduced by the pair $(\mathrm{G}, \varrho)$ is called a marked graph $\mathrm{G}$ marked by the set $X$ through the map $\varrho$ (see $[6,9]$ for the origins and applications). Note that (by abuse of language) we may introduce the corresponding marked graph as $\mathrm{G}\left(x_{1}, x_{2}, \ldots, x_{k}\right)$ when the definition of $\varrho$ (especially its range) is clear from the context (for examples see Figures $3(a)$ and $3(b)$ ). Also, (by abuse of language) we may refer to the vertex $x_{i}$ as the vertex $\varrho\left(x_{i}\right) \in V(\mathrm{G})$. This is most natural when $X \subseteq V(\mathrm{G})$ and vertices in $X$ are marked by the corresponding elements in $V(\mathrm{G})$ through the identity mapping.

If $\varsigma: X \longrightarrow Y$ is a (not necessarily one-to-one) map, then one can obtain a new marked graph $(\mathrm{H}, \tau: Y \longrightarrow V(\mathrm{H}))$ by considering the pushout of the diagram

$$
\mathrm{Y} \stackrel{\varsigma}{\longleftarrow} \mathrm{X} \stackrel{\varrho}{\longrightarrow} \mathrm{G}
$$


in the category of graphs. It is well-known that in Grph the pushout exists and is a monomorphism (e.g. Figure 3(c) presents such a pushout (i.e. amalgam) of marked graphs $3(a)$ and $3(b))$. Also, it is easy to see that the new marked graph $(\mathrm{H}, \tau)$ can be obtained from $(\mathrm{G}, \varrho)$ by identifying the vertices in each inverse-image of $\varsigma$. Hence, again we may denote $(\mathrm{H}, \tau)$ as $\mathrm{G}\left(\varsigma\left(x_{1}\right), \varsigma\left(x_{2}\right), \ldots, \varsigma\left(x_{k}\right)\right)$, where we allow repetition in the list appearing in the brackets. Note that, with this notation, one may interpret $x_{i}$ 's as a set of variables in the graph structure $\mathrm{G}\left(x_{1}, x_{2}, \ldots, x_{k}\right)$, such that when one assigns other (new and not necessarily distinct) values to these variables one can obtain some other graphs (by identification of vertices) (e.g. see Figure $3(d)$ ).

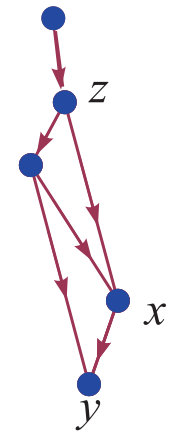

(a)

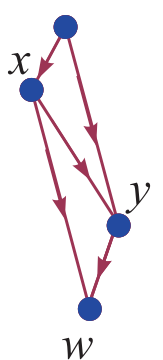

(b)

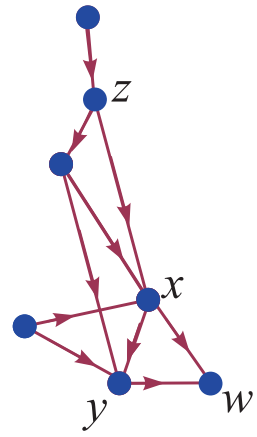

(c)

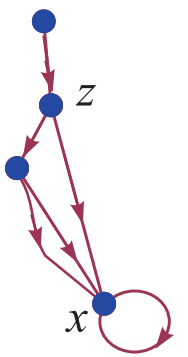

(d)

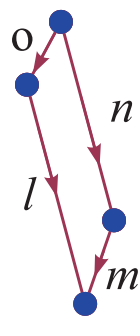

$(e)$

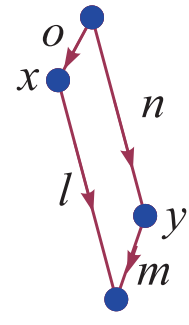

$(f)$

Figure 3: (a) A marked graph $\mathrm{G}(x, y, z)$, (b) A marked graph $\mathrm{H}(x, y, w)$, (c) The amalgamation $\mathrm{G}(x, y, z)+\mathrm{H}(x, y, w),(d)$ The marked graph $\mathrm{G}(x, x, z),(e)$ A labeled graph, $(f)$ A labeled marked graph.

On the other hand, given two marked graphs $(\mathrm{G}, \varrho)$ and $(\mathrm{H}, \tau)$ with

$$
X=\left\{x_{1}, x_{2}, \ldots, x_{k}\right\} \quad \text { and } \quad Y=\left\{y_{1}, y_{2}, \ldots, y_{l}\right\} \text {, }
$$

one can construct their amalgam

$$
(\mathrm{G}, \varrho)+_{\mathrm{S}}(\mathrm{H}, \tau)
$$

by forming the pushout of the following diagram,

$$
\mathrm{H} \stackrel{\tilde{\tau}}{\longleftarrow} \mathrm{S} \stackrel{\tilde{\varrho}}{\longrightarrow} \mathrm{G},
$$

in which $\mathrm{S}$ is a graph structure induced on $X \cap Y$ indicating the way edges should be identified in this amalgam such that one can define the extensions $\left.\tilde{\tau} \stackrel{\text { def }}{=} \tau\right|_{X \cap Y}$ and $\left.\tilde{\varrho} \stackrel{\text { def }}{=} \varrho\right|_{X \cap Y}$ as graph homomorphisms ${ }^{3}$. Following our previous notations we may denote the new structure by

$$
\mathrm{G}\left(x_{1}, x_{2}, \ldots, x_{k}\right)+\mathrm{H}\left(y_{1}, y_{2}, \ldots, y_{l}\right) .
$$

\footnotetext{
${ }^{3}$ Note that pushouts exist in the category of graphs and can be constructed as pushouts of the vertex set and the edge set naturally. For more details see the Appendix of [7].
} 
If there is no confusion about the definition of mappings (in what follows the maps $\tilde{\tau}$ and $\tilde{\varrho}$ are embeddings of a subgraph on $X \cap Y)$. Note that when $X \cap Y$ is the empty set, then the amalgam is the disjoint union of the two marked graphs. Also, by the universal property of the pushout diagram, the amalgam can be considered as marked graphs marked by $X$, $Y, X \cup Y$ or $X \cap Y$.

Sometimes it is preferred to partition the list of variables in a graph structure as,

$$
\mathrm{G}\left(x_{1}, x_{2}, \ldots, x_{k}, y_{1}, y_{2}, \ldots, y_{l}, z_{1}, z_{2}, \ldots, z_{m}\right) \text {. }
$$

In these cases we may either use bold symbols for an ordered list of variables and write this graph structure as $\mathrm{G}(\mathbf{x} ; \mathbf{y} ; \mathbf{z})$ (if there is no confusion about the size of the lists), or we may simply write

$$
\mathrm{G}\left(x_{1}, x_{2}, \ldots, x_{k} ; y_{1}, y_{2}, \ldots, y_{l} ; z_{1}, z_{2}, \ldots, z_{m}\right) \text {. }
$$

It is understood that a repeated appearance of a graph structure in an expression as $\mathrm{G}(v)+\mathrm{G}(v, w)$ is always considered as different isomorphic copies of the structure marked properly by the indicated labels (e.g. $\mathrm{G}(v)+\mathrm{G}(v, w)$ is an amalgam constructed by two different isomorphic copies of $G$ identified on the vertex $v$ where the vertex $w$ in one of these copies is marked).

\section{Definition 2. Labeled graphs}

An $\ell_{\mathrm{G}}$-graph $\mathrm{G}$ labeled by the set $L_{\mathrm{G}}$ consists of the data $\left(\mathrm{G}, \ell_{\mathrm{G}}: E(\mathrm{G}) \longrightarrow L_{\mathrm{G}}\right)$, where $\mathrm{G}$ is a graph and $\ell_{\mathrm{G}}$ is the labeling map (e.g. see Figure $3(e)$ ). A (labeled graph) homomorphism $\left(\sigma_{V}, \sigma_{E}, \sigma_{\ell}\right)$ from an $\ell_{\mathrm{G}}$-graph $\mathrm{G}=\left(V(\mathrm{G}), E(\mathrm{G}), \ell_{\mathrm{G}}\right)$ to an $\ell_{\mathrm{H}}$-graph $\mathrm{H}=$ $\left(V(\mathrm{H}), E(\mathrm{H}), \ell_{\mathrm{H}}\right)$ is a triple

$$
\sigma_{V}: V(\mathrm{G}) \longrightarrow V(\mathrm{H}), \quad \sigma_{E}: E(\mathrm{G}) \longrightarrow E(\mathrm{H}) \quad \text { and } \quad \sigma_{\ell}: L_{\mathrm{G}} \longrightarrow L_{\mathrm{H}}
$$

where all maps are compatible with the structure maps, i.e., conditions of Equation 1 are satisfied as well as,

$$
\ell_{\mathrm{H}} \circ \sigma_{E}=\sigma_{\ell} \circ \ell_{\mathrm{G}}
$$

The set of homomorphisms from the $\ell_{\mathrm{G}}$-graph $\mathrm{G}$ to the $\ell_{\mathrm{H}}$-graph $\mathrm{H}$ is denoted by $\operatorname{Hom}_{\ell}(\mathrm{G}, \mathrm{H})$, and LGrph stands for the category of labeled graphs and their homomorphisms.

It is obvious that the space of all graphs can be identified with the class of all labeled graphs, labeled by the one element set $\{1\}$. Hence, we treat all graphs labeled by a set of just one element, as ordinary (i.e. not labeled) graphs. Also, it is instructive to note that the concept of a labeled graph and the category LGrph has a central importance in the whole world of combinatorial objects (e.g. see [17]).

Accordingly, a labeled marked graph $(\mathrm{G}, \varrho, \ell)$, in which vertices are marked by the set $X$ and the edges are labeled by the set $L$, consists of the data

$$
(\mathrm{G}, \varrho: X \hookrightarrow V(\mathrm{G}), \ell: E(\mathrm{G}) \longrightarrow L),
$$


where $(\mathrm{G}, \varrho)$ is a marked $\ell$-graph equipped with the labeling $\ell: E(\mathrm{G}) \longrightarrow L$ of its edges (e.g. see Figure $3(f))$. Again, given two labeled marked graphs

$$
\left(\mathrm{G}, \varrho_{\mathrm{G}}: X \hookrightarrow V(\mathrm{G}), \ell_{\mathrm{G}}: E(\mathrm{G}) \longrightarrow L_{\mathrm{G}}\right) \text { and }\left(\mathrm{H}, \varrho_{\mathrm{H}}: Y \hookrightarrow V(\mathrm{H}), \ell_{\mathrm{H}}: E(\mathrm{H}) \longrightarrow L_{\mathrm{H}}\right),
$$

one may consider these graphs as $L_{\mathrm{G}} \cup L_{\mathrm{H}}$-labeled marked graphs and consider the pushout of Diagram 3 in LGrph, which exists when, intuitively, the $L_{\mathrm{G}} \cup L_{\mathrm{H}}$-labeled marked graph obtained by identifying vertices in $X \cap Y$ can be constructed by assigning the common labels of the overlapping edges in the intersection (i.e. the labels of overlapping edges must be compatible). This graph amalgam will be denoted in a concise form (when there is no confusion) by

$$
\mathrm{G}\left(\varrho_{\mathrm{G}}(X)\right)+\mathrm{H}\left(\varrho_{\mathrm{H}}(Y)\right) .
$$

Also, it is instructive to add some notes about automorphisms of labeled graphs. First, it is clear that given an automorphism $\left(\sigma_{V}, \sigma_{E}\right)$ of a labeled graph $\mathrm{G}$ on $k$ vertices then we have $\sigma_{V} \in \mathbf{S}_{\mathbf{k}}$. On the other hand, a permutation $\gamma \in \mathbf{S}_{\mathbf{k}}$ is said to induce an automorphism of $\mathrm{G}$ if there exists a map $\sigma_{E}$ on the edge-set such that $\left(\gamma, \sigma_{E}\right)$ is an automorphism of G. In particular, for reduced graphs if $\gamma \in \mathbf{S}_{\mathbf{k}}$ induces an automorphism then that automorphism is the unique automorphism induced by $\gamma$.

It is also important to note that given $\gamma \in \mathbf{S}_{\mathbf{k}}$ one may identify $\mathrm{G}$ along with any one of its permuted versions determined by an induced automorphism through $\gamma$ and again obtain a copy of $\mathrm{G}$ itself (hence this identification process is independent of the choice of the automorphism induced by $\gamma$ up to isomorphism.).

\subsection{Cylinders and the category of $(\Gamma, m)$-raphs}

\section{Definition 3. Cylinders}

A cylinder $\mathrm{C}(\mathbf{y}, \mathbf{z}, \epsilon)$ of thickness $(t, k)$ (or a $(t, k)$-cylinder for short), with the initial base $\mathrm{B}^{-}$and the terminal base $\mathrm{B}^{+}$(see Figure $4(a)$ ), is a labeled marked graph with the following data,

- A labeled marked graph $\mathrm{C}(\mathbf{y}, \mathbf{z})$.

- An ordered list $\mathbf{y}=\left(y_{1}, y_{2}, \ldots, y_{k}\right)$ of $k$ distinct elements with $0<k$ such that

$$
\left\{y_{1}, y_{2}, \ldots, y_{k}\right\} \subseteq V(\mathrm{C}), \quad \text { and } \quad \mathrm{B}^{-} \stackrel{\text { def }}{=} \mathrm{C}\left[y_{1}, y_{2}, \ldots, y_{k}\right] \text {. }
$$

- An ordered list $\mathbf{z}=\left(z_{1}, z_{2}, \ldots, z_{k}\right)$ of $k$ distinct elements with $0<k$ such that

$$
\left\{z_{1}, z_{2}, \ldots, z_{k}\right\} \subseteq V(\mathrm{C}), \quad \text { and } \quad \mathrm{B}^{+} \stackrel{\text { def }}{=} \mathrm{C}\left[z_{1}, z_{2}, \ldots, z_{k}\right] .
$$

- A one to one partial function $\epsilon: \widehat{1 \ldots k} \rightarrow \widehat{1 \ldots k}$, with

$$
|\operatorname{domain}(\epsilon)|=|\operatorname{range}(\epsilon)|=t,
$$

indicating equality between $t$ elements of $\mathbf{y}$ and $\mathbf{z}$, in the sense that

$$
\epsilon(i)=j \Leftrightarrow y_{i}=z_{j}
$$


- The mapping $y_{i} \mapsto z_{i}(1 \leqslant i \leqslant k)$ induces an isomorphism of the bases $\mathrm{B}^{-}$and $\mathrm{B}^{+}$ as labeled graphs.

Hereafter, we always refer to $\epsilon$ as a relation, i.e. $\epsilon=\{(i, \epsilon(i)): i \in \operatorname{domain}(\epsilon)\}$. Also, note that in general a cylinder is a directed graph and also has an intrinsic direction itself from $\mathrm{B}^{-}$to $\mathrm{B}^{+}$. In this regard the cylinder $-\mathrm{C}$ refers to the cylinder which is identical to $\mathrm{C}$ but with a reversed intrinsic direction (i.e. the bases are interchanged).

Moreover, a cylinder $\mathrm{C}(\mathbf{y}, \mathbf{z}, \epsilon)$ is said to be bilaterally symmetric (or bsymmetric for short) if there exists an automorphism of $\mathrm{C}$ as $\left(\sigma_{V}, \sigma_{E}\right): \mathrm{C} \longrightarrow \mathrm{C}$ that maps $\mathrm{B}^{-}$to $\mathrm{B}^{+}$ isomorphically as marked graphs, such that,

- $\forall i \in \widehat{1 \ldots k} \sigma\left(y_{i}\right)=z_{i}$, and $\sigma\left(z_{i}\right)=y_{i}$,

- $\sigma\left(\mathrm{B}^{-}\right)=\mathrm{B}^{+}, \quad \sigma\left(\mathrm{B}^{+}\right)=\mathrm{B}^{-}($as labeled graphs $)$,

Also, we partition the vertex set of a $(t, k)$-cylinder into the base vertices that appear in $V\left(\mathrm{~B}^{-}\right) \cup V\left(\mathrm{~B}^{+}\right)$, and the inner vertices that do not appear on either base. A $(0, k)$ cylinder whose bases are empty graphs and has no inner vertex is called a plain cylinder. A generalized loop cylinder is a cylinder for which $\mathrm{B}^{-}=\mathrm{B}^{+}$.

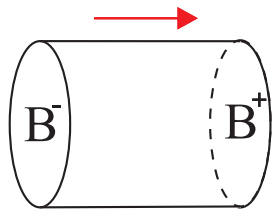

(a)

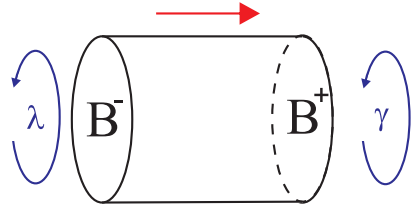

(b)

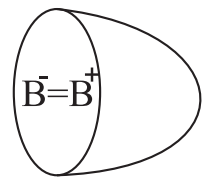

(c)

Figure 4: (a) A general form of a cylinder, (b) A cylinder with a twist $(\lambda, \gamma),(c)$ A generalized loop cylinder.

A Pultr template (for digraphs) is a quadruple $\tau=\left(\mathrm{B}, \mathrm{C}, \eta_{1}, \eta_{2}\right)$, where $\mathrm{B}$ and $\mathrm{C}$ are digraphs and $\eta_{1}, \eta_{2}: \mathrm{B} \rightarrow \mathrm{C}$ are digraph homomorphisms ${ }^{4}$. As described in the Section 1 , note that Pultr's left adjoint functor $-\otimes \tau$ acting on a digraph $\mathrm{G}$ gives rise to a digraph $\mathrm{G} \otimes \tau$ that can be constructed by taking a copy of the template $\tau_{e}$ for each edge $e$ of $\mathrm{G}$ and then identifying the corresponding bases for each vertex of $\mathrm{G}$. It must be emphasized that in this procedure the central role is played by the induced subgraphs $\eta_{1}(\mathrm{~B})$ and $\eta_{2}(\mathrm{~B})$ in C. On the other hand, Pultr's right adjoint construction acting on a digraph $\mathrm{H}$ produces a digraph whose set of vertices consists of all digraph homomorphisms $\xi: \mathrm{B} \longrightarrow \mathrm{H}$ and there is a directed edge from $\xi_{1}$ to $\xi_{2}$ if there exists a homomorphism $\rho: \mathrm{C} \longrightarrow \mathrm{H}$ such that $\xi_{i}=\rho \circ \eta_{i}(i \in\{1,2\})$. Again, in this setting too, the central role is played by the induced subgraphs $\xi_{i}(\mathrm{~B})=\rho\left(\eta_{i}(\mathrm{~B})\right)$ which are forced to be identified for all vertices with at least one in-going and one out-going edges. Hence, it is deduced that the full power of the constructions are essentially reflected in the case when $\eta_{i}$ is an isomorphism on its range (i.e. when $\eta_{1}(\mathrm{~B}) \simeq \eta_{2}(\mathrm{~B}) \simeq \mathrm{B}$ ). Also, note that if one let $\mathrm{B}$ be an empty graph

\footnotetext{
${ }^{4}$ Note that this is essentially a cospan of graphs.
} 
on $k$ vertices then this can be considered as a marking of the induced subgraph in the range of $\eta_{i}$. These observations justifies Definition 3 and referring to such templates as cylinders. It is also instructive to note that applying automorphisms of bases as twists (see Definition 5) will also force to define bases as induced subgraphs, at least to capture the most important and generic case of the construction ${ }^{5}$.

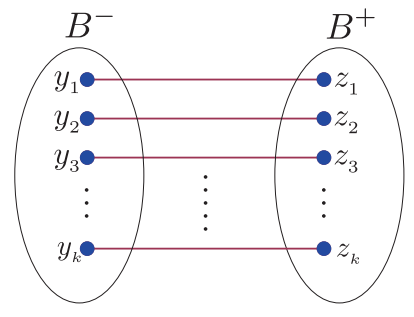

(a)

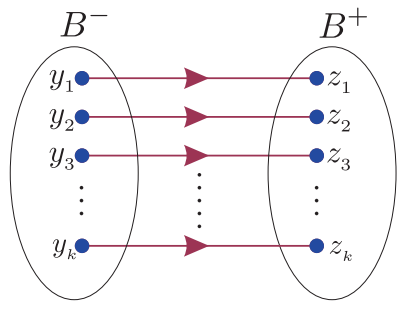

(b)

Figure 5: (a) The identity cylinder $\mathrm{I}_{k}(\mathbf{y}, \mathbf{z})$ (see Examples 4 and 18), (b) The directed identity cylinder $\overrightarrow{\mathrm{I}}_{k}(\mathbf{y}, \mathbf{z})$.

Example 4. In this example we illustrate a couple of cylinders to clarify the definition.

\section{- The identity cylinder, $\mathrm{I}_{k}(\mathrm{y}, \mathrm{z})$}

Define the identity cylinder on $2 k$ vertices as the plain cylinder $\mathrm{I}_{k}(\mathbf{y}, \mathbf{z})$, where both bases are empty graphs on $k$ vertices and the only edges are $y_{i} z_{i}$ for $i \in \widehat{1 \ldots k}$ (see Figure 5(a)). Clearly, we may define $\mathrm{I}(y, z) \stackrel{\text { def }}{=} \mathrm{I}_{1}(y, z)$ (or I for short) standing for an edge. Also, we use the notation $\overrightarrow{\mathrm{I}}_{k}(\mathbf{y}, \mathbf{z})$ for the directed version, where edges are directed from $\mathrm{B}^{-}$to $\mathrm{B}^{+}$(see Figure $5(\mathrm{~b})$ ).

- The $\sqcap$-cylinder, $\sqcap(\mathbf{y}, \mathbf{z})$

This is a $(0,2)$-cylinder (or a 2-cylinder for short) that is depicted in Figure 6(a), for which $\epsilon=\emptyset$. This cylinder will be used in the sequel to construct the Petersen and generalized Petersen graphs (see Example 18).

- The path cylinder, $\mathrm{P}_{n}(y, z)$

For $n>0$ this is a 1-cylinder with $n+1$ vertices that is depicted in Figure 6(b), forming a path of length $n$. This cylinder will be used in the sequel to construct subdivision and fractional powers of a graph (see Examples 17 and 20).

Analogously, one may define directed path cylinders $\overrightarrow{\mathrm{P}}_{n}(y, z)$ (see Figure $6(\mathrm{c})$ ).

\section{- The looped line-graph cylinder, $\wedge(\mathbf{y}, \mathbf{z})$}

\footnotetext{
${ }^{5}$ It is not hard to verify that simple generalizations of the proposed definition is possible that essentially will not give rise to any interesting application as far as we are aware.
} 


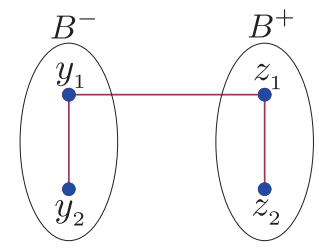

(a)

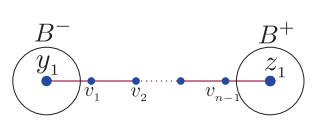

(b)

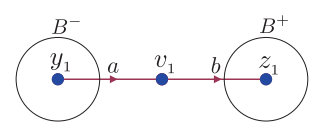

$(c)$

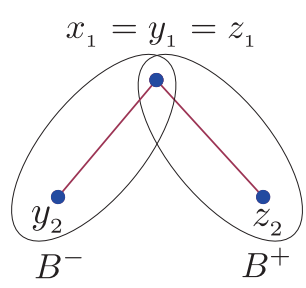

(d)

Figure 6: (a) The $\sqcap$-cylinder, (b) The path cylinder $\mathrm{P}_{n},(c)$ The directed path cylinder $\overrightarrow{\mathrm{P}}_{2}$ of length two, $(d)$ The looped line-graph cylinder.

This is a $(1,2)$-cylinder on 3 vertices, depicted in Figure 6(d), for which $\epsilon=\{(1,1)\}$ (i.e. $y_{1}=z_{1}$ ). This cylinder will be used in the sequel to construct looped line-graphs (see Example 20).

\section{Definition 5. Twist of a cylinder}

An ordered pair $(\gamma, \lambda) \in A u t_{\ell}\left(\mathrm{B}^{-}\right) \times A u t_{\ell}\left(\mathrm{B}^{+}\right)$is said to be a twist of a cylinder $\mathrm{C}(\mathbf{y}, \mathbf{z}, \epsilon)$, where $A u t_{\ell}$ stands for the group of labeled graph automorphisms. Note that if $|\mathbf{y}|=k$, then $\left(\gamma_{V}, \lambda_{V}\right) \in \mathbf{S}_{\mathbf{k}} \times \mathbf{S}_{\mathbf{k}}$ (see Figure $4(b)$ and note that the bases $\mathrm{B}^{-}$and $\mathrm{B}^{+}$are isomorphic by definition). Hereafter, we may omit the subscripts for the maps $\lambda_{V}$ or $\lambda_{E}$ when the map is clear by the element on which it acts.

A twisted cylinder $\mathrm{C}\left(\mathbf{y}^{\prime}, \mathbf{z}^{\prime},(\gamma, \lambda) \epsilon\right)$, as the result of action of a twist $(\gamma, \lambda)$ on $\mathrm{C}(\mathbf{y}, \mathbf{z}, \epsilon)$, is a cylinder for which we have $\mathbf{y}^{\prime}=\mathbf{y} \gamma, \mathbf{z}^{\prime}=\mathbf{z} \lambda$ and $(\gamma, \lambda) \stackrel{\text { def }}{=}\{(\gamma(i), \lambda(j)):(i, j) \in \epsilon\}$. Note that by definition the bases of a twisted cylinder are isomorphic to the corresponding bases of the original cylinder.

A twist $(\gamma, \lambda)$ is said to be reflexive if $\gamma=\lambda$.

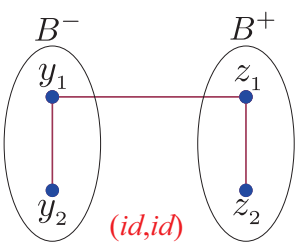

(a)

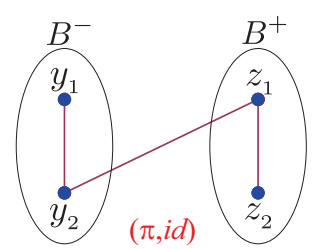

(b)

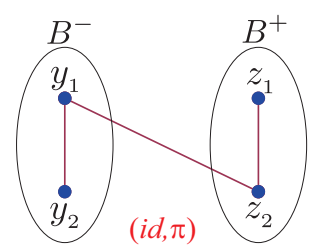

$(c)$

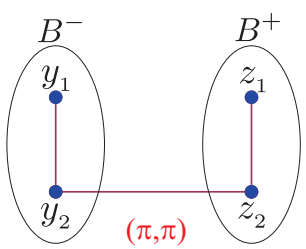

$(d)$

Figure 7: Twists of the $\sqcap$-cylinder (see Example 6).

\section{Example 6. Twists of $\sqcap(y, z)$}

Setting $\pi \stackrel{\text { def }}{=}\lfloor 2,1\rceil$, each element of $\mathbf{S}_{\mathbf{2}} \times \mathbf{S}_{\mathbf{2}} \simeq\{(i d, i d),(i d, \pi),(\pi, i d),(\pi, \pi)\}$ induces a unique automorphism of an edge and satisfies the conditions of Definition 5, and consequently, by abuse of language, may be reffered to as a twist of the $\sqcap$-cylinder depicted in Figure 6(a) (see Figure 7). 


\section{Definition 7. A $\Gamma$-coherent set of cylinders}

Given integers $t$ and $k$ and a subgroup $\Gamma \leqslant \mathbf{S}_{\mathbf{k}}$, then a set of $(t, k)$-cylinders

$$
\mathrm{C}=\left\{\mathrm{C}^{j}\left(\mathbf{y}^{j}, \mathbf{z}^{j}, \epsilon^{j}\right) \mid j \in \widehat{1 \ldots m}\right\}
$$

is said to be $\Gamma$-coherent, if

- For all $j, r \in \widehat{1 \ldots m}$, the mapping $y_{i}^{j} \mapsto y_{i}^{r}(i \in \widehat{1 \ldots k})$ from $\mathrm{B}^{-}\left(\mathrm{C}^{j}\right)$ to $\mathrm{B}^{-}\left(\mathrm{C}^{r}\right)$ induces an isomorphism of these bases as labeled graphs,

- Each $\gamma \in \Gamma$ induces an automorphism of $\mathrm{B}^{-}\left(\mathrm{C}^{1}\right)$.

A $\Gamma$-coherent set of cylinders is said to be bsymmetric if each one of its cylinders is a bsymmetric cylinder.

Again, when $m=1$, we may forget about the set and talk about the $\Gamma$-cylinder $\mathrm{C}(\mathbf{y}, \mathbf{z}, \epsilon)$ itself.

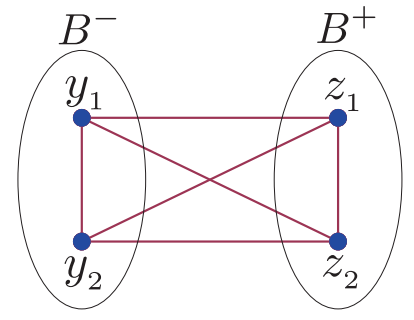

$(a)$

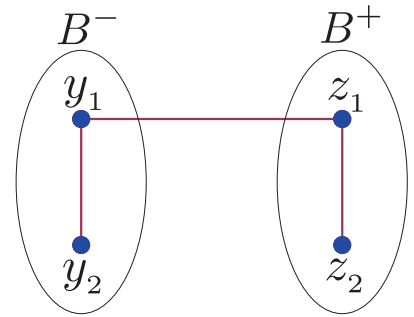

(b)

Figure 8: A set of $\mathbf{S}_{2}$-coherent cylinders.

\section{Example 8.}

- Every set of 1-cylinders is $\mathbf{S}_{\mathbf{1}}$-coherent. For instance, every set of path cylinders is $\mathbf{S}_{1}$-coherent.

- The $\sqcap$-cylinder and the cylinder shown in Figure $8(a)$ form a $\mathbf{S}_{\mathbf{2}}$-coherent set of cylinders.

Note that if a set of $(t, k)$-cylinders is a $\mathbf{S}_{k}$-coherent set, then either all bases are isomorphic to the complete graph $\mathrm{K}_{k}$ or they are isomorphic to the empty graph $\overline{\mathrm{K}}_{k}$.

\section{Definition 9. The category of $(\Gamma, m)$-graphs}

Given a subgroup $\Gamma \leqslant \mathbf{S}_{\mathbf{k}}$, a $(\Gamma, m)$-graph is a graph labeled by the set

$$
L_{\Gamma, m} \stackrel{\text { def }}{=}\left\{\left(\gamma^{-}, i, \gamma^{+}\right) \quad \mid \quad i \in \widehat{1 \ldots m}, \quad \gamma^{-}, \gamma^{+} \in \Gamma\right\} .
$$

Note that in this setting each label as $\left(\gamma^{-}, i, \gamma^{+}\right)$encodes the type of the cylinder in $i$ and the corresponding twists are induced by $\left(\gamma^{-}, \gamma^{+}\right)$. 
Explicitly, consider an edge $e \in E(\mathrm{G})$ in a $(\Gamma, m)$-graph $\mathrm{G}$ with $e^{-}=u$ and $e^{+}=v$. Then if $\ell_{\mathrm{G}}$ is the labeling map, and $\ell_{\mathrm{G}}(e)=\left(\gamma^{-}, i, \gamma^{+}\right)$, we use the following notations

$$
\begin{gathered}
\ell_{\mathrm{G}}^{*}(e) \stackrel{\text { def }}{=} i . \\
\ell_{\mathrm{G}}^{-}(e) \stackrel{\text { def }}{=} \gamma^{-}, \quad \ell_{\mathrm{G}}^{+}(e) \stackrel{\text { def }}{=} \gamma^{+},
\end{gathered}
$$

where by definition $\gamma^{-}$is the action corresponding to $e^{-}=u$ and $\gamma^{+}$is the action corresponding to $e^{+}=v$. Also, we may use the notations

$$
\ell_{\mathrm{G}}^{u}(e) \stackrel{\text { def }}{=} \ell_{\mathrm{G}}^{-}(e)=\gamma^{-}, \quad \ell_{\mathrm{G}}^{v}(e) \stackrel{\text { def }}{=} \ell_{\mathrm{G}}^{+}(e)=\gamma^{+} .
$$

If $e_{1}, e_{2} \in E(\mathrm{G})$ are incident edges, where $e_{1}^{a}=e_{2}^{b}=w$ for $a, b \in\{-,+\}\left(\right.$ e.g. $\left.e_{1}^{-}=e_{2}^{+}\right)$, then we define a label difference function $\Delta_{\mathrm{G}}^{a b}$ as

$$
\Delta_{\mathrm{G}}^{a b}\left(e_{1}, e_{2}\right) \stackrel{\text { def }}{=}\left[\ell_{\mathrm{G}}^{a}\left(e_{1}\right)\right]^{-1}\left[\ell_{\mathrm{G}}^{b}\left(e_{2}\right)\right] .
$$

Of course, when there is not any ambiguity we may omit the subscripts or superscripts.

Let $\mathrm{G}$ and $\mathrm{H}$ be two $(\Gamma, m)$-graphs labeled by the maps $\ell_{\mathrm{G}}$ and $\ell_{\mathrm{H}}$, respectively. Then a $(\Gamma, m)$-homomorphism from $\mathrm{G}$ to $\mathrm{H}$ is a graph homomorphism $\left(\sigma_{V}, \sigma_{E}\right): \mathrm{G} \longrightarrow \mathrm{H}$ such that

$$
\ell_{\mathrm{G}}^{*}=\ell_{\mathrm{H}}^{*} \circ \sigma_{\mathrm{E}}
$$

and for any pair of edges $e_{1}, e_{2} \in E(\mathrm{G})$ that intersect in $e_{1}^{a}=e_{2}^{b}(a, b \in\{-,+\})$,

$$
\Delta_{\mathrm{G}}^{a b}\left(e_{1}, e_{2}\right)=\Delta_{\mathrm{H}}^{a b}\left(\sigma_{E}\left(e_{1}\right), \sigma_{E}\left(e_{2}\right)\right),
$$

or equivalently,

$$
\left[\ell_{\mathrm{G}}^{a}\left(e_{1}\right)\right]^{-1}\left[\ell_{\mathrm{G}}^{b}\left(e_{2}\right)\right]=\left[\ell_{\mathrm{H}}^{a}\left(\sigma_{E}\left(e_{1}\right)\right)\right]^{-1}\left[\ell_{\mathrm{H}}^{b}\left(\sigma_{E}\left(e_{2}\right)\right)\right] .
$$

The set of homomorphisms from the $(\Gamma, m)$-graph $\mathrm{G}$ to the $(\Gamma, m)$-graph $\mathrm{H}$ is denoted by $\operatorname{Hom}_{\Gamma, m}(\mathrm{G}, \mathrm{H})$, and $\mathbf{L G r p h}(\Gamma, m)$ stands for the category of $(\Gamma, m)$-graphs and their homomorphisms. Note that when $m=1$ and $\Gamma=\mathbf{i d}$ then $\mathbf{G r p h} \simeq \mathbf{L G r p h}\left(\mathbf{S}_{1}, 1\right)$.

In what follows, we may exclude variables in our notations when they are set to their default (i.e. trivial) values. As an example, note that $\mathrm{C}(\mathbf{y}, \mathbf{z})$ is used when $\epsilon$ is empty (i.e. $t=0)$ and we may refer to it as a $k$-cylinder. Similarly, we may talk about $\Gamma$-graphs when $m=1$.

Also, it is clear that one may enumerate any finite set of cylinders, and consequently, when there is no ambiguity, we may refer to cylinders by their name instead of their number.

Proposition 10. Let $\mathrm{G}$ and $\mathrm{H}$ be two $(\Gamma, m)$-graphs and $\left(\sigma_{V}, \sigma_{E}\right)$ be a graph homomorphism. Then the following conditions are equivalent.

a) The pair $\left(\sigma_{V}, \sigma_{E}\right)$ is a $(\Gamma, m)$-homomorphism. In other words, for any pair of two edges $e_{1}, e_{2} \in E(\mathrm{G})$ that intersect at a vertex $e_{1}^{a}=e_{2}^{b}($ for $a, b \in\{-,+\})$,

$$
\Delta_{\mathrm{G}}^{a b}\left(e_{1}, e_{2}\right)=\Delta_{\mathrm{H}}^{a b}\left(\sigma_{E}\left(e_{1}\right), \sigma_{E}\left(e_{2}\right)\right) .
$$


b) For any vertex $u \in V(\mathrm{G})$, there exists a unique constant $\alpha_{u} \in \Gamma$ such that for any edge $e \in E(\mathrm{G})$ that intersects in $e^{a}=u($ for $a \in\{-,+\})$, we have,

$$
\ell_{\mathrm{G}}^{a}(e)=\alpha_{u}\left[\ell_{\mathrm{H}}^{a}\left(\sigma_{E}(e)\right)\right]
$$

Proof. $(\mathrm{a} \Rightarrow \mathrm{b})$ Fix a vertex $u$ and an edge $e_{0}$ with $e_{0}^{+}=u$ (one can do the same with the assumption $e_{0}^{-}=u$ in a similar way) and define,

$$
\alpha_{u} \stackrel{\text { def }}{=} \ell_{\mathrm{G}}^{+}\left(e_{0}\right)\left[\ell_{\mathrm{H}}^{+}\left(\sigma_{E}\left(e_{0}\right)\right)\right]^{-1} .
$$

Then, for any other edge $e$ with $e^{a}=u$ for $a \in\{+,-\}$ we have,

$$
\begin{aligned}
\ell_{\mathrm{G}}^{a}(e) & =\ell_{\mathrm{G}}^{+}\left(e_{0}\right)\left[\ell_{\mathrm{G}}^{+}\left(e_{0}\right)\right]^{-1} \ell_{\mathrm{G}}^{a}(e) \\
& =\ell_{\mathrm{G}}^{+}\left(e_{0}\right)\left[\ell_{\mathrm{H}}^{+}\left(\sigma_{E}\left(e_{0}\right)\right)\right]^{-1}\left[\ell_{\mathrm{H}}^{a}\left(\sigma_{E}(e)\right)\right] \\
& =\alpha_{u}\left[\ell_{\mathrm{H}}^{a}\left(\sigma_{E}(e)\right)\right] .
\end{aligned}
$$

$(\mathrm{b} \Rightarrow \mathrm{a})$ is clear by definition of the constant $\alpha_{u}$.

Definition 11. Consider a graph $\mathrm{G}$ labeled by the set $L_{\Gamma, m}$ and the map $\ell_{\mathrm{G}}$, along with a vector $\alpha \in \Gamma^{|V(G)|}$. Then, we define the labeled graph $\mathrm{G}_{\alpha}$ on the base graph of $\mathrm{G}$ with the labeling

$$
\ell_{\mathrm{G}_{\alpha}}(e)=\left(\alpha_{u} \ell_{\mathrm{G}}^{-}(e), \ell_{\mathrm{G}}^{*}(e), \alpha_{v} \ell_{\mathrm{G}}^{+}(e)\right), \quad \text { where } e^{-}=u \text { and } e^{+}=v .
$$

Note that if $\mathrm{C}=\left\{\mathrm{C}^{j}\left(\mathbf{y}^{j}, \mathbf{y}^{j}, \epsilon^{j}\right) \mid j \in \widehat{1 . . m}\right\}$ is a $\Gamma$-coherent set of cylinders labeled by $L_{\Gamma, m}$, then for any vector $\alpha \in \Gamma^{|V(\mathrm{G})|}$,

$$
\mathrm{C}_{\alpha} \stackrel{\text { def }}{=}\left\{\mathrm{C}_{\alpha}^{j}\left(\mathbf{y}^{j}, \mathbf{z}^{j}, \epsilon^{j}\right) \mid j \in \widehat{1 \ldots m}\right\}
$$

is also a $\Gamma$-coherent set of cylinders. The following results is a direct consequence of Proposition 10.

Corollary 12. Let $\mathrm{G}$ and $\mathrm{H}$ be two graphs labeled by the set $L_{\Gamma, m}$ and the maps $\ell_{\mathrm{G}}$ and $\ell_{\mathrm{H}}$, respectively. Then, considering these graphs as objects of the category of labeled graphs $\mathbf{L G r p h}$, and also as objects of the category of $(\Gamma, m)$-graphs $\mathbf{L} \mathbf{G r p h}(\Gamma, m)$, we have

$$
\exists \alpha \in \Gamma^{|V(\mathrm{G})|} \quad \operatorname{Hom}_{\Gamma, \mathrm{m}}(\mathrm{G}, \mathrm{H}) \neq \emptyset \Leftrightarrow \operatorname{Hom}_{\ell}\left(\mathrm{G}, \mathrm{H}_{\alpha}\right) \neq \emptyset \quad \Leftrightarrow \quad \operatorname{Hom}_{\ell}\left(\mathrm{G}_{\alpha^{-1}}, \mathrm{H}\right) \neq \emptyset .
$$

\section{Definition 13. Reflexive and symmetric $(\Gamma, m)$-graphs}

A $(\Gamma, m)$-label $(\gamma, i, \lambda)$ is said to reflexive if $\gamma=\lambda$. Also, we say that a $(\Gamma, m)$-graph is reflexive if all of its labels are reflexive.

On the other hand, a $(\Gamma, m)$-graph $\mathrm{G}$ is said to be symmetric if its edges can be partitioned into dual pairs as $e$ and $\tilde{e}$ such that 


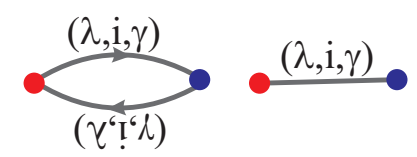

Figure 9: Symmetry in $(\Gamma, m)$-graphs.

- $e^{-}=\tilde{e}^{+}=u$ and $e^{+}=\tilde{e}^{-}=v$,

- If $\ell_{\mathrm{G}}(e)=(\gamma, i, \lambda)$ for some $\gamma, \lambda \in \Gamma$, then $\ell_{\mathrm{G}}(\tilde{e})=(\lambda, i, \gamma)$.

Note that the data contained in a symmetric $(\Gamma, m)$-graph can be encoded in a corresponding undirected graph on the same vertex set and simple edges as $u v$ with labels $(\gamma, i, \lambda)$ since actions correspond only to vertices (see Figure 9).

Lemma 14. Let $\sigma \in \operatorname{Hom}_{\Gamma, \mathrm{m}}(\mathrm{G}, \mathrm{H})$, and also $\ell_{\mathrm{G}}$ and $\ell_{\mathrm{H}}$ be the $(\Gamma, m)$-labelings of $\mathrm{G}$ and $\mathrm{H}$ respectively.

1. If $e_{1}, e_{2} \in E(\mathrm{G}), e_{1}^{-}=e_{2}^{-}, e_{1}^{+}=e_{2}^{+}$and $\ell_{\mathrm{H}}\left(\sigma_{E}\left(e_{1}\right)\right)=\ell_{\mathrm{H}}\left(\sigma_{E}\left(e_{2}\right)\right)$ then $\ell_{\mathrm{G}}\left(e_{1}\right)=$ $\ell_{\mathrm{G}}\left(e_{2}\right)$.

2. If $\mathrm{G}$ is reflexive and $\alpha_{u}$ given by Proposition 10 is a constant function on vertices, then the image of $\sigma$ is a reflexive subgraph of $\mathrm{H}$.

Proof.

1. Let $e_{1}^{-}=e_{2}^{-}=u$ and $e_{1}^{+}=e_{2}^{+}=v$, then by Proposition 10 for some $\alpha_{u}$ we have

$$
\ell_{\mathrm{G}}^{-}\left(e_{1}\right)=\alpha_{u} \ell_{\mathrm{H}}^{-}\left(\sigma_{E}\left(e_{1}\right)\right)=\alpha_{u} \ell_{\mathrm{H}}^{-}\left(\sigma_{E}\left(e_{2}\right)\right)=\ell_{\mathrm{G}}^{-}\left(e_{2}\right) .
$$

The proof of $\ell_{\mathrm{G}}^{v}\left(e_{1}\right)=\ell_{\mathrm{G}}^{v}\left(e_{2}\right)$ is similar.

2. Let $e^{-}=u$ and $e^{+}=v$. By the hypothesis, there exist $\lambda \in \Gamma$ and $i \in \widehat{1 \ldots m}$ such that $\ell_{\mathrm{G}}(e)=(\lambda, i, \lambda)$. Consequently, since $\alpha_{u}=\alpha_{v}$, we have $\ell_{\mathrm{H}}\left(\sigma_{E}(e)\right)=$ $\left(\alpha_{u} \lambda, i, \alpha_{u} \lambda\right)$.

\section{Definition 15. Reduced $(\Gamma, m)$-graphs}

There is a subtlety about the concept of the edge reduced subgraph of a $(\Gamma, m)$-graphs that deserves emphasis.

Note that in a directed $(\Gamma, m)$-graph two edges are similar if they have the same initial and terminal vertices as well as the same label. In particular, two directed loops on a vertex $v$ with labels $(\gamma, i, \lambda)$ and $(\lambda, i, \gamma)$, respectively, are a dual pair of edges which are not similar. Hence, such pairs of loops should not be identified to construct the edge reduced subgraph.

However, on the other hand, two undirected loops on a vertex $v$ with labels $(\gamma, i, \lambda)$ and $(\lambda, i, \gamma)$, respectively, are similar by means of symmetry and should be identified in an edge reduced subgraph.

These facts are crucial in our definition for the exponential graph in the next section. 


\section{The cylindrical graph construction}

This section presents the main construction in this article and its dual. In more detail, given a $(\Gamma, m)$-graph, $\mathrm{G}$, and a $\Gamma$-coherent set of cylinders, one may think of a new graph constructed by replacing each edge of $\mathrm{G}$ by a twisted version of the cylinder determined by the label of the edge. In this regard, the main point is that the label determines the cylinder as well as two permutations which are compatible to the structure of the bases of all cylinders by definitions, and consequently, this construction is independent of the choice of induced automorphisms as twists (up to isomorphism) determined by the labels of the edges of $\mathrm{G}$.

The dual construction is essentially a $(\Gamma, m)$-graph that is constructed as a graph on the set of homomorphisms from cylinders whose structure will be explained in Section 3.2.

\subsection{The cylindrical construction}

Definition 16. Let $\mathrm{C} \stackrel{\text { def }}{=}\left\{\mathrm{C}^{j}\left(\mathbf{y}^{j}, \mathbf{z}^{j}, \epsilon^{j}\right) \mid j \in \widehat{1 . . . m}\right\}$ be a set of $\Gamma$-coherent $(t, k)$ cylinders, where each $\mathrm{C}^{j}$ is an $\ell_{j}$-graph. Also, let $\mathrm{G}$ be a $(\Gamma, m)$-graph labeled by the map $\ell_{\mathrm{G}}$.

First, note that given a $(\Gamma, m)$-label $\left(\gamma^{-}, j, \gamma^{+}\right)$one may choose a twist $\left(\tilde{\gamma}^{-}, \tilde{\gamma}^{+}\right)$of $\mathrm{C}^{j}\left(\mathbf{y}^{j}, \mathbf{z}^{j}, \epsilon^{j}\right)$ where $\tilde{\gamma}^{-}$and $\tilde{\gamma}^{+}$are induced automorphisms through $\gamma^{-}$and $\gamma^{+}$, respectively. Then, in this setting, one may construct the twisted cylinder

$$
\mathrm{C}^{j}\left(\mathbf{y}^{j} \tilde{\gamma}^{-}, \mathbf{z}^{j} \tilde{\gamma}^{+},\left(\tilde{\gamma}^{-}, \tilde{\gamma}^{+}\right) \epsilon^{j}\right)
$$

whose bases are isomorphic to the corresponding bases of $\mathrm{C}^{j}\left(\mathbf{y}^{j}, \mathbf{z}^{j}, \epsilon^{j}\right)$. Moreover, the set of these twisted cylinders again constitute a $\Gamma$-coherent set of cylinders, regardless of the choices for the induced automorphisms $\tilde{\gamma}^{-}$and $\tilde{\gamma}^{+}$. Hence, by abuse of notation, we will refer to any one of such twisted cylinders by $\mathrm{C}^{j}\left(\mathbf{y}^{j} \gamma^{-}, \mathbf{z}^{j} \gamma^{+},\left(\gamma^{-}, \gamma^{+}\right) \epsilon^{j}\right)$ since such choices will not affect the pushout constructions that follow.

The cylindrical product of $\mathrm{G}$ and $\mathrm{C}$ is an amalgam that, intuitively, can be described as the graph constructed by replacing each edge of $\mathrm{G}$ whose label is $\left(\gamma^{-}, j, \gamma^{+}\right)$, by a copy of the cylinder $\mathrm{C}^{j}$ twisted by $\left(\gamma^{-}, \gamma^{+}\right)$, while we identify the corresponding bases of the twisted cylinders that intersect at the position of each vertex of G. Formally, this construction denoted by $\mathrm{G} \otimes_{\Gamma} \mathrm{C}$ can be defined as the following pushout construction,

$$
\mathrm{G} \bigotimes_{\Gamma} \mathrm{C} \stackrel{\text { def }}{=} \sum_{u v \in E(\mathrm{G}): \ell_{\mathrm{G}}(u v)=\left(\gamma^{u}, j, \gamma^{v}\right)} \mathrm{C}^{j}\left(\mathbf{u}^{j} \gamma^{u}, \mathbf{v}^{j} \gamma^{v},\left(\gamma^{u}, \gamma^{v}\right) \epsilon^{j}\right)
$$

To be more descriptive, let us describe the construction in an algorithmic way as follows. Let $V(\mathrm{G})=\left\{v_{1}, v_{2}, \ldots, v_{n}\right\}$. Then for a $\Gamma$-coherent set of $(t, k)$-cylinders, $\mathrm{C}$, the graph $G \otimes_{\Gamma} C$ is constructed on $G_{0}$ as the graph that is generated by the following algorithm,

1. Blowing up vertices: (This step is just for clarification and may be omitted.) 
Consider the empty graph $\mathrm{G}_{0}$ with

$$
V\left(\mathrm{G}_{0}\right) \stackrel{\text { def }}{=} \bigcup_{j=1}^{n}\left\{v_{1}^{j}, v_{2}^{j}, \ldots, v_{k}^{j}\right\},
$$

and $E\left(\mathrm{G}_{0}\right)=\emptyset$.

2. Choosing the cylinders: For every edge $e \in E(\mathrm{G})$ with $e^{-}=p, e^{+}=q$ and the label $\ell_{\mathrm{G}}(e)=\left(\gamma^{-}, j, \gamma^{+}\right)$, choose a twisted cylinder $\mathrm{C}^{j}\left(\mathbf{y}^{j} \gamma^{-}, \mathbf{z}^{j} \gamma^{+},\left(\gamma^{-}, \gamma^{+}\right) \epsilon^{j}\right)$ and re-mark the vertices in each base, constructing the cylinder $\mathrm{C}^{j}\left(\mathbf{v}^{p}, \mathbf{v}^{q}\right)$ for which

$$
\begin{aligned}
& \text { - Let } \mathbf{v}^{p}=\mathbf{y}^{j} \gamma^{-}, \\
& \text {- Let } \mathbf{v}^{q}=\mathbf{z}^{j} \gamma^{+} .
\end{aligned}
$$

Consider the set $\mathrm{G}^{*}$ of disjoint union of all these cylinders constructed for each edge.

3. Identification: Consider the amalgam constructed on $\mathrm{G}_{0} \cup \mathrm{G}^{*}$ as a marked graph, identifying all vertices first with respect to markings, then with respect to $\epsilon^{j}$ 's and in the end, identify all edges corresponding to each other in different bases corresponding to a fixed vertex of $G$.

Note that given any edge $e \in E\left(\mathrm{G}_{\Gamma} \mathrm{C}\right)$, there exists an index $j \in \widehat{1 \ldots m}$ such that $e \in E\left(\mathrm{C}^{j}\right)$, and one may assign a well defined labeling

$$
\ell_{\mathrm{G}_{\Gamma} \mathrm{C}}(e) \stackrel{\text { def }}{=} \ell_{j}(e) .
$$

Consequently, $\mathrm{G} \otimes_{\Gamma} \mathrm{C}$ is an $\ell_{\mathrm{G} \otimes_{\Gamma} \mathrm{C}}$-graph.

Given $\mathrm{C}$ as a set of $\Gamma$-coherent bsymmetric $(t, k)$-cylinders, and an undirected graph $\mathrm{G}$, one may talk about the bsymmetric cylindrical construct $\mathrm{G}_{\Gamma}^{s} \mathrm{C}$ defined similarly through replacement of undirected edges by the corresponding cylinders. Note that $\mathrm{G}_{\Gamma}^{s} \mathrm{C}$ is essentially isomorphic to the construct $\overrightarrow{\mathrm{G}} \otimes_{\Gamma} \mathrm{C}$ where $\overrightarrow{\mathrm{G}}$ is the directed graph constructed from $\mathrm{G}$ by assigning directions to undirected edges arbitrarily. Also, if $\mathrm{C}$ is directed, then the bsymmetric construct $\mathrm{G} \otimes_{\Gamma}^{s} \mathrm{C}$ is also a directed graph although $\mathrm{G}$ is undirected. Clearly, $\tilde{G}$ is the corresponding symmetric graph for $G$, then $G \otimes_{\Gamma}^{s} \mathrm{C} \neq \tilde{\mathrm{G}} \otimes_{\Gamma} \mathrm{C}$. Moreover, following our notational abbreviations we may exclude the superscript $s$ when it is clear from the context that $\mathrm{G}$ is undirected and the construction is bsymmetric.

For a graph $\mathrm{G}$ with $V(\mathrm{G})=\left\{v_{1}, v_{2}, \ldots, v_{n}\right\}$, hereafter the vertex set of the cylindrical construct $\mathrm{G}_{\Gamma} \mathrm{C}$ is assumed to be $V\left(\mathrm{G}_{\Gamma} \mathrm{C}\right)=\bigcup_{j=1}^{m}\left\{v_{1}^{j}, v_{2}^{j}, \ldots, v_{k}^{j}\right\}$ where the superscript refers to the index of the corresponding vertex in $V(\mathrm{G})$ and, moreover, we may refer to the list $\mathbf{v}^{j} \stackrel{\text { def }}{=}\left(v_{1}^{j}, v_{2}^{j}, \ldots, v_{k}^{j}\right)$. Also, we assume that in each cylinder, the set of vertices that do not appear in the vertex sets of the bases are enumerated (say from 1 to $r$ ), and for each fixed edge $e \in E(\mathrm{G})$, we may refer to the list $\mathbf{w}^{e} \stackrel{\text { def }}{=}\left(w_{1}^{e}, w_{2}^{e}, \ldots, w_{r}^{e}\right)$ consisting of inner vertices that appear in $G \otimes_{\Gamma} \mathrm{C}$ when the edge $e$ is replaced by the corresponding cylinder.

In what follows we go through some examples to clarify the definition and its relation to other known constructions as well as some new ones related to the contents of Section 5 . 


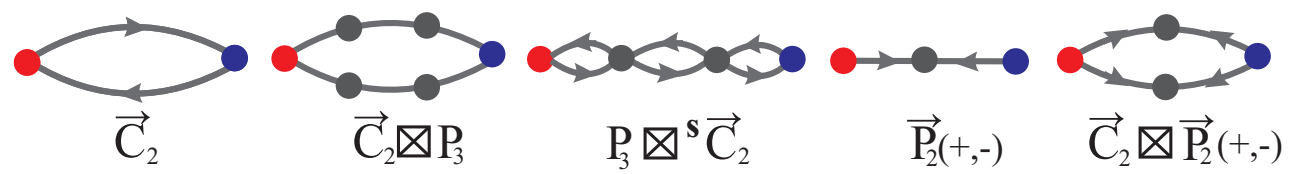

Figure 10: Different forms of cylindrical construction due to simplicity and symmetricalness properties of cylinders.

Example 17. It is clear that the identity cylinder satisfies the identity property $\mathrm{G} \otimes \mathrm{I}=\mathrm{G}$ in both directed and undirected cases (see Example 4). Note that, in general, cylindrical products using the identity cylinder gives rise to lifts of graphs (e.g. see [18] and references therein for the details, applications and background. Also see Figure 1.).

It is easy to see that $\mathrm{G} \otimes \mathrm{P}_{n}$ gives rise to an $n$-subdivision of $\mathrm{G}$ which can be described as subdividing every edge of $\mathrm{G}$ by $n-1$ vertices.

On the other hand, the cylindrical construction $\mathrm{G} \otimes \mathrm{C}$ for a 1-cylinder $\mathrm{C}(y, z)$ is the standard replacement operation (e.g. see [15] and references therein).

Some other standard graph constructions as different kinds of graph products, fiber construction, Pultr left adjoint construction and many other classical graph constructions are cylindrical (see [7] for more details).

Let us comment on some basic facts. The graph $\overrightarrow{\mathrm{P}}_{2}(+,-)$ depicted in Figure 10 shows that a bsymmetric cylinder may not be symmetric as a graph. Other graphs in Figure 10 show different variants of cylindrical construction when each component is directed or undirected. The case in which both components are undirected will be treated in Example 18.

One may also observe that the construct $G \otimes^{s} \vec{C}_{2}$ represents the functor that maps an undirected graph $\mathrm{G}$ to its corresponding symmetric graph (see Figure 10 and Section 4.2 for more on functorial properties).

Note that in this construction $\overrightarrow{\mathrm{C}}_{2}$ is a bsymmetric cylinder.

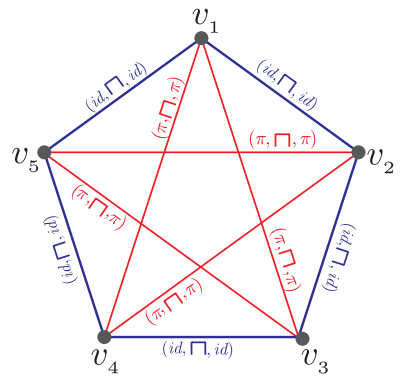

(a)

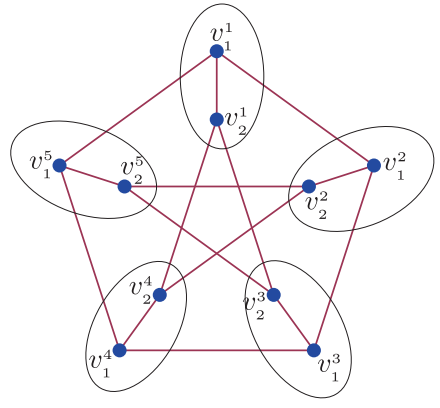

$(b)$

Figure 11: (a) The base graph $\mathrm{K}_{5}^{*}$, (b) The Petersen graph as $\mathrm{K}_{5}^{*} \bigotimes_{\mathbf{S}_{\mathbf{2}}} \sqcap$.

Example 18. In this example we elaborate on different descriptions of the Petersen graph using cylindrical constructions, where in this regard we will also describe any voltage graph 
construction as a special case of a cylindrical construction. This, in particular, shows that a graph may have many different descriptions as a cylindrical product.

\section{- Petersen graph and the $\sqcap$-cylinder}

Consider the complete graph $\mathrm{K}_{5}^{*}$ of Figure $11(a)$ as a $\mathbf{S}_{\mathbf{2}}$-graph, and note that the cylindrical construction $\left(\mathrm{K}_{5}^{*} \otimes_{\mathbf{S}_{\mathbf{2}}} \sqcap\right)$ gives rise to the Petersen graph as depicted in Figure 11(b).

\section{- Petersen graph and the voltage graph construction}

Let $A$ be a set of $k$ elements and $\Omega$ be a group that acts on $A$ from the right. Then it is clear that one may identify the set $A$ as $A=\left\{a_{1}, a_{2}, \ldots, a_{k}\right\}$ and $\Omega$ as a

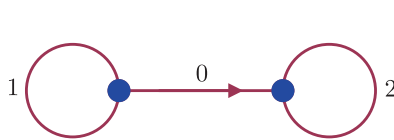

(a)

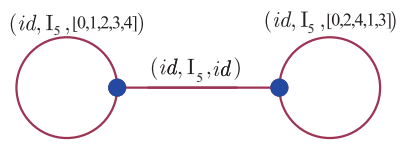

(b)

Figure 12: (a) Petersen Voltage graph, (b) The corresponding $(\Gamma, m)$-graph.

permutation group acting on the index set $\widehat{1 \ldots k}$.

An action voltage graph is a (directed) labeled graph $\left(\mathrm{G}, \ell_{\mathrm{G}}: E(\mathrm{G}) \longrightarrow \Omega\right.$ ) whose edges are labeled by the elements of the group $\Omega$. The derived graph of $\mathrm{G}$, denoted by $\tilde{\mathrm{G}}$, is a graph on the vertex set $V(\tilde{\mathrm{G}})=V(\mathrm{G}) \times A$, where there is an edge between $\left(u, a_{i}\right)$ and $\left(v, a_{j}\right)$ if $e=u v \in E(\mathrm{G})$ and $a_{j}=a_{i} \ell_{\mathrm{G}}(e)$.

When $\Omega$ acts on itself on the right, then $A=\Omega$ and the action voltage graph is simply called the voltage graph, when we usually refer to the corresponding derived graph as the voltage graph construct in this case. It is also easy to verify that Cayley graphs are among the well-known examples of voltage graphs (e.g. see [15] for more on voltage graphs and related topics).

Now, we show that derived graphs are cylindrical constructs. For this, given an action voltage graph $\left(\mathrm{G}, \ell_{\mathrm{G}}: E(\mathrm{G}) \longrightarrow \Omega\right.$ ), we define the corresponding $\Omega$-graph $\widehat{\mathrm{G}}$ as a graph with the same vertex and edge sets as $\mathrm{G}$, and the labeling

$$
\forall e \in E(\widehat{\mathrm{G}}) \quad \ell_{\widehat{\mathrm{G}}}(e)=\left(i d, \mathrm{I}_{k}, \rho\right) \Leftrightarrow \ell_{\mathrm{G}}(e)=\rho,
$$

where $\mathrm{I}_{k}$ is the identity cylinder on $k$ vertices (see Figure 5 ). Then it is easy to see that $\tilde{\mathrm{G}}=\widehat{\mathrm{G}} \otimes_{\Omega} \mathrm{I}_{k}$. Figure 12 shows the voltage graph and the corresponding $\mathbf{Z}_{5}$-graph to construct the Petersen graph using this construction.

Let $\sigma: \mathrm{G} \rightarrow \mathrm{H}$ be a vertex surjective homomorphism between reduced simple graphs. Then, it is clear by definitions that the inverse image of any vertex is an independent set. Let $k$ be the maximum size of these inverse images and note that the graph induced on any pair of such inverse images produces a plain $k$-cylinder when one may add extra isolated vertices to the bases if necessary. This shows that $G$ is the reduced part of a 
cylindrical construction by plain cylinders and one may deduce that cylindrical construction generalizes the concept of a covering or a homomorphic pre-image in a fairly broad sense.

\subsection{The exponential construction}

Given a $\Gamma$-coherent set of $(t, k)$-cylinders containing $m \geqslant 1$ cylinders, there is a canonical way of constructing an exponential graph that will be defined in the next definition.

Definition 19. The exponential graph $[\mathrm{C}, \mathrm{H}]_{\Gamma}$

For any given labeled graph $\mathrm{H}$ and a $\Gamma$-coherent set of $(t, k)$-cylinders

$$
\mathrm{C} \stackrel{\text { def }}{=}\left\{\mathrm{C}^{j}\left(\mathbf{y}^{j}, \mathbf{z}^{j}, \epsilon^{j}\right) \mid j \in \widehat{1 \ldots m}\right\}
$$

the exponential graph $[\mathrm{C}, \mathrm{H}]_{\Gamma}$ is defined (up to isomorphism) as a $(\Gamma, m)$-graph in the following way,

i) Consider the right action of $\Gamma$ on $V(\mathrm{H})^{k}$ as

$$
\mathbf{v} \gamma=\left(v_{1}, v_{2}, \ldots, v_{k}\right) \gamma \stackrel{\text { def }}{=}\left(v_{\gamma(1)}, v_{\gamma(2)}, \ldots, v_{\gamma(k)}\right),
$$

and the corresponding equivalence relation whose equivalence classes determine the orbits of this action, i.e.

$$
\mathbf{u} \sim_{\Gamma} \mathbf{v} \quad \Leftrightarrow \quad \exists \gamma \in \Gamma \quad \mathbf{u} \gamma=\mathbf{v}
$$

Also, fix a set of representatives $U$ of these equivalence classes and let

$$
V_{U}\left([\mathrm{C}, \mathrm{H}]_{\Gamma}^{*}\right) \stackrel{\text { def }}{=} U=\left\{\mathbf{u}_{1}, \mathbf{u}_{2}, \ldots, \mathbf{u}_{d}\right\}
$$

ii) There is an edge $e \stackrel{\text { def }}{=} \mathbf{u}_{i} \mathbf{u}_{k} \in E_{U}\left([\mathrm{C}, \mathrm{H}]_{\Gamma}^{*}\right)$ with the label $\ell(e)=\left(\gamma^{\mathbf{u}_{i}}, j, \gamma^{\mathbf{u}_{k}}\right)$ if and only if there exists a homomorphism

$$
\left(\sigma_{V}, \sigma_{E}\right) \in \operatorname{Hom}_{\ell}\left(\mathrm{C}^{j}\left(\mathbf{y}^{j}, \mathbf{z}^{j}, \epsilon^{j}\right), \mathrm{H}\right)
$$

such that,

$$
\sigma_{V}\left(\mathbf{y}^{j} \gamma^{\mathbf{u}_{i}}\right)=\mathbf{u}_{i} \quad \text { and } \quad \sigma_{V}\left(\mathbf{z}^{j} \gamma^{\mathbf{u}_{k}}\right)=\mathbf{u}_{k} .
$$

iii) Define $[\mathrm{C}, \mathrm{H}]_{\Gamma}$ to be the reduced graph $[\mathrm{C}, \mathrm{H}]_{\Gamma}=\operatorname{red}\left([\mathrm{C}, \mathrm{H}]_{\Gamma}^{*}\right)$.

Using Proposition 10, one may verify that for two different sets of representatives $U$ and $W$ we have

$$
\forall i \in \widehat{1 \ldots d} \quad \exists \gamma_{i} \in \Gamma \quad \mathbf{u}_{i} \gamma_{i}=\mathbf{w}_{i}
$$

and that the map $\sigma=\left(\sigma_{V}, \sigma_{E}\right)$ defined as

$$
\forall i \in \widehat{1 \ldots d} \sigma_{V}\left(\mathbf{u}_{i}\right) \stackrel{\text { def }}{=} \mathbf{w}_{i},
$$


and for $e=\mathbf{u}_{i} \mathbf{u}_{k}$ with $\ell(e)=\left(\gamma^{\mathbf{u}_{i}}, j, \gamma^{\mathbf{u}_{k}}\right)$,

$$
\sigma_{E}(e) \stackrel{\text { def }}{=} \mathbf{w}_{i} \mathbf{w}_{k}, \quad \text { with } \quad \ell\left(\sigma_{E}(e)\right)=\left(\gamma_{i} \gamma^{\mathbf{u}_{i}}, j, \gamma_{k} \gamma^{\mathbf{u}_{k}}\right)
$$

is an isomorphism of $(\Gamma, m)$-graphs, and consequently, hereafter, we assume that every exponential graph is constructed with respect to a fixed class of representatives $U$, and we omit the corresponding subscript since we are just dealing with such graphs up to an isomorphism. The representative $\mathbf{u}_{i}$ of the equivalence class $[\mathbf{u}]_{\sim_{\Gamma}}$ (where $\mathbf{u}_{i}=\mathbf{u} \gamma$ for some $\gamma \in \Gamma)$ is denoted by $\langle\mathbf{u}\rangle_{\Gamma}$.

An exponential graph by definition is a reduced directed graph, however, if one is dealing with a $\Gamma$-coherent set of bsymmetric $(t, k)$-cylinders then the exponential graph is clearly symmetric, and consequently, one may also talk about the corresponding undirected symmetric exponential graph, $[\mathrm{C}, \mathrm{H}]_{\Gamma}^{s}$, in which all edges are simple. Also, note that $[\mathrm{C}, \mathrm{H}]_{\Gamma}$ is defined either when both $\mathrm{C}$ and $\mathrm{H}$ are directed graphs or when both $\mathrm{C}$ and $\mathrm{H}$ are undirected graphs.

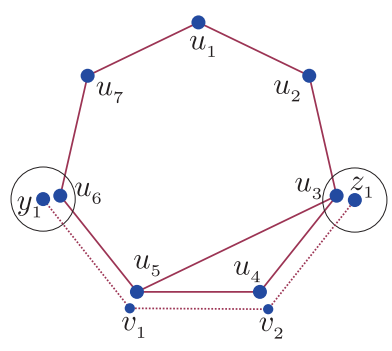

(a)

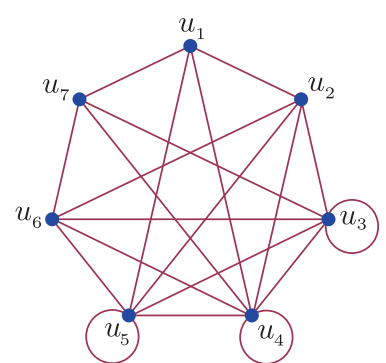

(b)

Figure 13: (a) A base graph $\mathrm{G}$ and the cylinder $\mathrm{P}_{3}$, (b) The exponential graph $\mathrm{G}^{3}=$ $\left[\mathrm{P}_{3}, \mathrm{G}\right]^{s}$.

Example 20. It is clear that the identity cylinder satisfies the identity property $[\mathrm{I}, \mathrm{H}]=\mathrm{H}$ in both directed and symmetric cases (see Example 4).

The exponential graph $[\mathrm{C}, \mathrm{H}]$ for a 1-cylinder $\mathrm{C}(y, z)$ is the standard indicator construction (e.g. see [15] and references therein), where the undirected case $[\mathrm{C}, \mathrm{H}]^{s}$ is again the standard construction through the corresponding automorphism (see Definition 3).

It is easy to check that the $n$th power ${ }^{6} \mathrm{G}^{n}$ of a simple graph $\mathrm{G}$, which is defined to be the undirected graph on the vertex set $V\left(\mathrm{G}^{n}\right) \stackrel{\text { def }}{=} V(\mathrm{G})$, obtained by adding an edge $u v$ if there exists a walk of length $n$ in $\mathrm{G}$ starting at $u$ and ending at $v$, can be described as follows,

$$
\mathrm{G}^{n}=\left[\mathrm{P}_{n}, \mathrm{G}\right]^{s} .
$$

Note that $\mathrm{G}^{n}$ contains loops when $n$ is even.

\footnotetext{
${ }^{6}$ Note that this is different from the other definition for the $n$th power of a graph, where an edge is added when there exists a walk of length less than or equal to $n$.
} 
Clearly, $\mathrm{G} \otimes \mathrm{P}_{n}$ gives rise to an $n$-subdivision of $\mathrm{G}$ which can be described as subdividing every edge of $\mathrm{G}$ by $n-1$ vertices. Also, note that one may describe the fractional power $\mathrm{G}^{\frac{m}{n}}$ as follows

$$
\mathrm{G}^{\frac{m}{n}} \stackrel{\text { def }}{=}\left[\mathrm{P}_{m}, \mathrm{G} \otimes \mathrm{P}_{n}\right]^{s} .
$$

(See [14] for the definition and more details. Also, see [7] for more on closures and openings.)

\section{Example 21.}

\section{- A directed example}

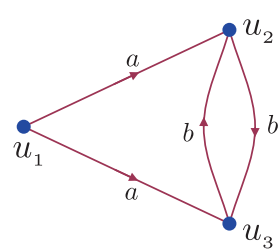

(a)

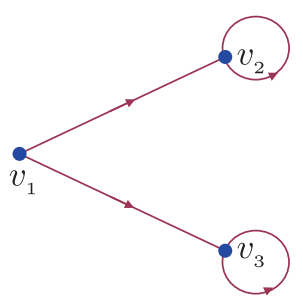

(b)

Figure 14: (a) The directed graph T, (b) The graph $\left[\overrightarrow{\mathrm{P}}_{2}, \mathrm{~T}\right]$.

Figure 14 shows a directed graph $\mathrm{T}$ and the exponential graph $\left[\overrightarrow{\mathrm{P}}_{2}, \mathrm{~T}\right]$ (here note that we have excluded all default values of the parameters involved in our notations).
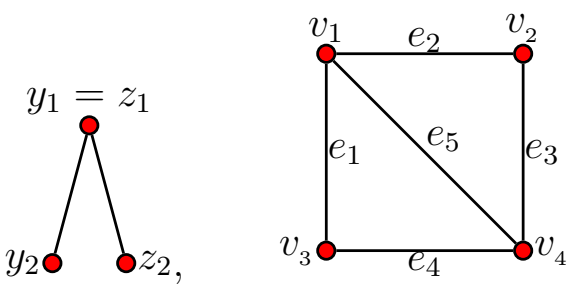

Figure 15: The looped line-graph cylinder $\wedge$ and the base graph $\mathrm{H}$.

- The looped line-graph ([19], also see Proposition 26),

It can be verified that the looped line-graph of a given graph $\mathrm{H}$ can be described as the exponential graph $[\wedge, \mathrm{H}]_{\mathbf{S}_{2}}^{s}$. Note that in this case, there is an extra loop on each vertex of the line-graph.

Let us go through the details of such a construction for the graph $\mathrm{H}$ depicted in Figure 15 .

1. First, we present the set of vertices corresponding to the equivalence classes (see Figure 16(a)). 


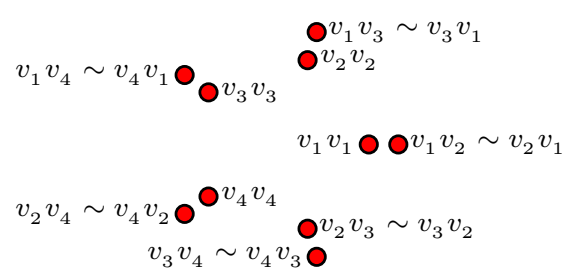

$(a)$

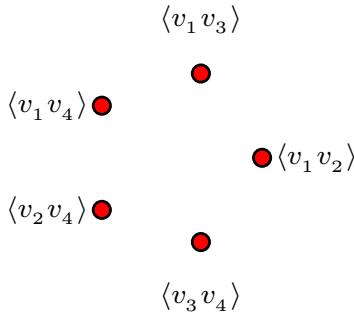

(b)

Figure 16: (a) Arranging vertices, (b) Fixing representatives for $[\wedge, \mathrm{H}]_{\mathrm{S}_{2}}^{s}$.

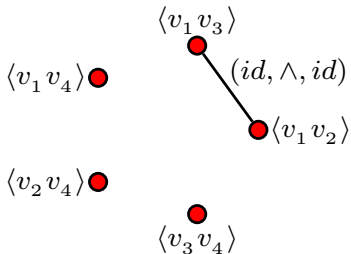

(a)

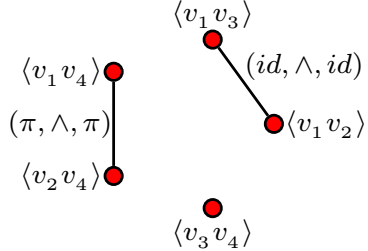

(b)

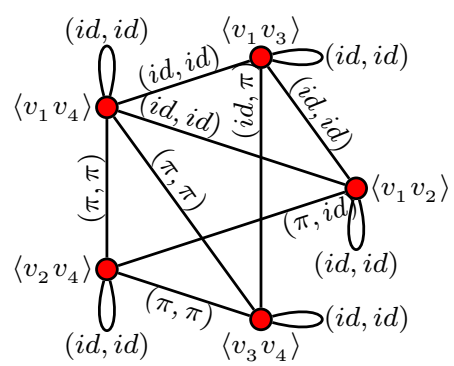

(c)

Figure 17: $(a, b)$ Some edges of graph $[\wedge, \mathrm{H}]_{\mathbf{S}_{2}}^{s}(c)$ The exponential graph $[\wedge, \mathrm{H}]_{\mathbf{S}_{2}}^{s}$.

2. Fix a set of representatives. Exclude isolated vertices i.e. delete any pair $v_{i} v_{j}$ for which we can not find a graph homomorphism $\mathrm{K}_{2} \cong \mathrm{B}^{-} \rightarrow \mathrm{H}\left(\left[v_{i}, v_{j}\right]\right.$ ) (see Figure 16(b)).

3. To specify the edges,

- Consider a graph homomorphism $\wedge \rightarrow \mathrm{H}$ for which $y_{1}=z_{1} \mapsto v_{1}, \quad y_{2} \mapsto$ $v_{2}$, and $\quad z_{2} \mapsto v_{3}$. Note that this homomorphism corresponds to the edge $\left\langle v_{1} v_{3}\right\rangle-\left\langle v_{1} v_{2}\right\rangle$ as depicted in Figure 17(a).

- Again, consider a graph homomorphism for which $y_{1} \mapsto v_{4}, \quad y_{2} \mapsto v_{2}$, and $z_{2} \mapsto v_{1}$, and consequently, we have the edge $\left\langle v_{4} v_{1}\right\rangle-\left\langle v_{4} v_{2}\right\rangle$, however, we have to use the twist $\pi=\lfloor 1,2\rceil$ on both bases to find the corresponding representatives as $\pi\left(v_{4} v_{1}\right)=v_{1} v_{4}$ and $\pi\left(v_{4} v_{2}\right)=v_{2} v_{4}$ (see Figure 17(b)).

- By continuing this procedure, we obtain the loop-lined graph as depicted in Figure 17.

\section{Main duality theorem}

In this section we state our main result stating a duality between the exponential and cylindrical constructions. Consequences and remarks will proceed the proof. 


\subsection{The fundamental duality}

\section{Theorem 22. The fundamental duality}

Let $\mathrm{C}=\left\{\mathrm{C}^{j}\left(\mathbf{y}^{j}, \mathbf{z}^{j}, \epsilon^{j}\right) \mid j \in \widehat{1 \ldots m}\right\}$ be a $\Gamma$-coherent set of $(t, k)$-cylinders, and also let $\mathrm{G}$ be a non-empty $(\Gamma, m)$-graph. Then for any labeled graph $\mathrm{H}$,

a) $\operatorname{Hom}_{\ell}\left(\mathrm{G} \otimes_{\Gamma} \mathrm{C}, \mathrm{H}\right) \neq \emptyset \Leftrightarrow \operatorname{Hom}_{\Gamma, \mathrm{m}}\left(\mathrm{G},[\mathrm{C}, \mathrm{H}]_{\Gamma}\right) \neq \emptyset$.

b) There exist a section

$$
s_{\mathrm{G}, \mathrm{H}}: \operatorname{Hom}_{\Gamma, \mathrm{m}}\left(\mathrm{G},[\mathrm{C}, \mathrm{H}]_{\Gamma}^{*}\right) \rightarrow \operatorname{Hom}_{\ell}\left(\mathrm{G} \otimes_{\Gamma} \mathrm{C}, \mathrm{H}\right) .
$$

c) There is a retraction

$$
r_{\mathrm{G}, \mathrm{H}}: \operatorname{Hom}_{\ell}\left(\mathrm{G} \bigotimes_{\Gamma} \mathrm{C}, \mathrm{H}\right) \rightarrow \operatorname{Hom}_{\Gamma, \mathrm{m}}\left(\mathrm{G},[\mathrm{C}, \mathrm{H}]_{\Gamma}^{*}\right),
$$

such that $r_{\mathrm{G}, \mathrm{H}} \circ s_{\mathrm{G}, \mathrm{H}}=\mathbf{1}_{\operatorname{Hom}_{\ell}\left(\mathrm{G} \otimes_{\Gamma} \mathrm{C}, \mathrm{H}\right)}$, where $\mathbf{1}_{\mathrm{Hom}_{\ell}\left(\mathrm{G} \otimes_{\Gamma} \mathrm{C}, \mathrm{H}\right)}$ is the identity mapping.
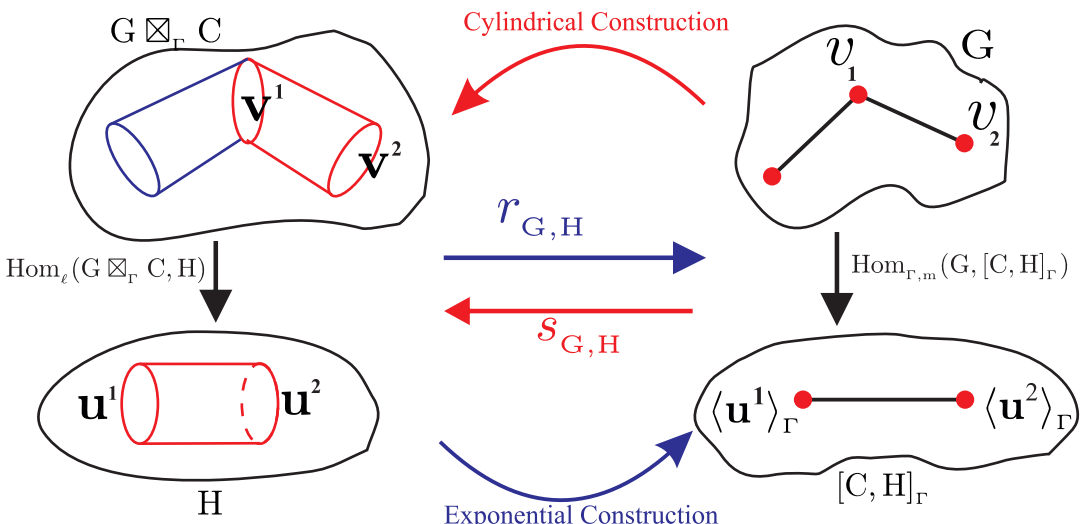

Figure 18: Schematic diagram for the proof of Theorem 22.

Proof.

First, note that since $[\mathrm{C}, \mathrm{H}]_{\Gamma}$ is the reduced part of $[\mathrm{C}, \mathrm{H}]_{\Gamma}^{*}$, the set $\operatorname{Hom}_{\Gamma, \mathrm{m}}\left(\mathrm{G},[\mathrm{C}, \mathrm{H}]_{\Gamma}^{*}\right)$ is empty, if and only if the set $\operatorname{Hom}_{\Gamma, \mathrm{m}}\left(\mathrm{G},[\mathrm{C}, \mathrm{H}]_{\Gamma}\right)$ is empty.

$((a) \Rightarrow)$ If $\operatorname{Hom}_{\ell}\left(G \nabla_{\Gamma} \mathrm{C}, \mathrm{H}\right) \neq \emptyset$, then there exists a homomorphism

$$
\sigma=\left(\sigma_{V}, \sigma_{E}\right) \in \operatorname{Hom}_{\ell}\left(\mathrm{G} \otimes_{\Gamma} \mathrm{C}, \mathrm{H}\right) .
$$

Assume that $V(\mathrm{G})=\left\{v_{1}, v_{2}, \ldots, v_{n}\right\}$ and

$$
\forall i \in \widehat{1 \ldots n}, \quad \mathbf{u}^{i} \stackrel{\text { def }}{=}\left(\sigma_{V}\left(v_{1}^{i}\right), \sigma_{V}\left(v_{2}^{i}\right), \ldots, \sigma_{V}\left(v_{k}^{i}\right)\right),
$$

where $\mathbf{v}^{i}=\left(v_{1}^{i}, v_{2}^{i}, \ldots, v_{k}^{i}\right)$ is the set of vertices that blow up at $v_{i}$ in $\mathrm{G} \nabla_{\Gamma} \mathrm{C}$. Now, define $r_{\mathrm{G}, \mathrm{H}}\left(\left(\sigma_{V}, \sigma_{E}\right)\right)=\left(\sigma_{V}^{\prime}, \sigma_{E}^{\prime}\right)$, where $\sigma_{V}^{\prime}: V(\mathrm{G}) \longrightarrow V\left([\mathrm{C}, \mathrm{H}]_{\Gamma}^{*}\right)$ and

$$
\forall i \in \widehat{1 \ldots n} \quad \sigma_{V}^{\prime}\left(v_{i}\right) \stackrel{\text { def }}{=}\left\langle\mathbf{u}^{i}\right\rangle_{\Gamma} .
$$


Note that by the definition of the exponential graph construction, for each $i \in \widehat{1 \ldots n}$, there exists a unique $\alpha_{i} \in \Gamma$ such that $\left\langle\mathbf{u}^{i}\right\rangle_{\Gamma}=\mathbf{u}^{i} \alpha_{i}$, hence we have

$$
\forall i \in \widehat{1 \ldots n} \quad \sigma_{V}^{\prime}\left(v_{i}\right) \stackrel{\text { def }}{=} \mathbf{u}^{i} \alpha_{i} .
$$

Again, for each $e \in E(\mathrm{G})$ with $\iota(e)=v_{i}, \tau(e)=v_{j}$ and $\ell_{\mathrm{G}}(e)=\left(\gamma^{-}, t, \gamma^{+}\right)$, define,

$$
\sigma_{E}^{\prime}(e) \stackrel{\text { def }}{=} e^{\prime}=\left\langle\mathbf{u}^{i}\right\rangle\left\langle\mathbf{u}^{j}\right\rangle
$$

such that

$$
\iota\left(e^{\prime}\right)=\left\langle\mathbf{u}^{i}\right\rangle_{\Gamma}=\mathbf{u}^{i} \alpha_{i}, \quad \tau\left(e^{\prime}\right)=\left\langle\mathbf{u}^{j}\right\rangle_{\Gamma}=\mathbf{u}^{j} \alpha_{j}, \quad \ell_{[\mathrm{C}, \mathrm{H}]_{\Gamma}^{*}}\left(e^{\prime}\right)=\left(\alpha_{i} \gamma^{-}, t, \alpha_{j} \gamma^{+}\right) .
$$

Now, we show that the mapping $\sigma^{\prime}=\left(\sigma_{V}^{\prime}, \sigma_{E}^{\prime}\right)$ is well defined and is a $(\Gamma, m)$ homomorphism in $\operatorname{Hom}_{\Gamma, \mathrm{m}}\left(\mathrm{G},[\mathrm{C}, \mathrm{H}]_{\Gamma}^{*}\right)$.

First, note that since $\ell_{\mathrm{G}}(e)=\left(\gamma^{-}, t, \gamma^{+}\right)$, by cylindrical construction, the restriction of $\sigma$ to the cylinder on this edge $e=v_{i} v_{j}$, gives a homomorphism

$$
\sigma^{e}=\left(\sigma_{V}^{e}, \sigma_{E}^{e}\right) \in \operatorname{Hom}_{\ell}\left(\mathrm{C}^{t}\left(\mathbf{y}^{t} \gamma^{-}, \mathbf{z}^{t} \gamma^{+},\left(\gamma^{-}, \gamma^{+}\right) \epsilon^{t}\right), \mathrm{H}\right)
$$

such that

$$
\sigma_{V}^{e}\left(\mathbf{y}^{t} \gamma^{-}\right)=\mathbf{u}^{i} \quad \text { and } \quad \sigma_{V}^{e}\left(\mathbf{z}^{t} \gamma^{+}\right)=\mathbf{u}^{j}
$$

Hence, by applying the twists $\left(\alpha_{i}, \alpha_{j}\right)$ we obtain a new homomorphism

$$
\tilde{\sigma}^{e}=\left(\tilde{\sigma}_{V}^{e}, \tilde{\sigma}_{E}^{e}\right) \in \operatorname{Hom}_{\ell}\left(\mathrm{C}^{t}\left(\mathbf{y}^{t} \alpha_{i} \gamma^{-}, \mathbf{z}^{t} \alpha_{j} \gamma^{+},\left(\gamma^{-}, \gamma^{+}\right) \epsilon^{t}\right), \mathrm{H}\right)
$$

such that

$$
\tilde{\sigma}_{V}^{e}\left(\mathbf{y}^{t} \alpha_{i} \gamma^{-}\right)=\mathbf{u}^{i} \alpha_{i}=\left\langle\mathbf{u}^{i}\right\rangle_{\Gamma}, \tilde{\sigma}_{V}^{e}\left(\mathbf{z}^{t} \alpha_{j} \gamma^{+}\right)=\mathbf{u}^{j} \alpha_{j}=\left\langle\mathbf{u}^{j}\right\rangle_{\Gamma},
$$

and by definition of the exponential graph, this shows that there is an edge

$$
\tilde{e} \stackrel{\text { def }}{=}\left\langle\mathbf{u}^{i}\right\rangle_{\Gamma}\left\langle\mathbf{u}^{j}\right\rangle_{\Gamma} \in E\left([\mathrm{C}, \mathrm{H}]_{\Gamma}^{*}\right)
$$

with the label $\ell_{[\mathrm{C}, \mathrm{H}]_{\Gamma}^{*}}(\tilde{e})=\left(\alpha_{i} \gamma^{-}, t, \alpha_{j} \gamma^{+}\right)$. This shows that $\sigma^{\prime}$ is a graph homomorphism. Also, by Proposition 10, it is clear that

$$
\sigma^{\prime}=\left(\sigma_{V}^{\prime}, \sigma_{E}^{\prime}\right) \in \operatorname{Hom}_{\Gamma, \mathrm{m}}\left(\mathrm{G},[\mathrm{C}, \mathrm{H}]_{\Gamma}^{*}\right) .
$$

$((a) \Leftarrow)$ Fix a homomorphism $\sigma^{\prime}=\left(\sigma_{V}^{\prime}, \sigma_{E}^{\prime}\right) \in \operatorname{Hom}_{\Gamma, \mathrm{m}}\left(\mathrm{G},[\mathrm{C}, \mathrm{H}]_{\Gamma}^{*}\right)$ and assume that

$$
\forall i \in \widehat{1 \ldots n} \quad \sigma_{V}^{\prime}\left(v_{i}\right)=\left\langle\mathbf{u}^{i}\right\rangle_{\Gamma} \in V\left([\mathrm{C}, \mathrm{H}]_{\Gamma}^{*}\right) \text {. }
$$


Also, by Proposition 10, we know that for each $i \in \widehat{1 \ldots n}$ there exists $\alpha_{i} \in \Gamma$ such that for any edge $e$ intersecting $v_{i}$ we have,

$$
\ell_{\mathrm{G}}^{v_{i}}(e)=\alpha_{i}\left[\ell_{[\mathrm{C}, \mathrm{H}]_{\Gamma}^{*}}^{\left\langle\mathbf{u}^{i}\right\rangle_{\Gamma}}\left(\sigma_{E}^{\prime}(e)\right)\right] .
$$

Again, fix an edge $e=v_{i} v_{j} \in E(\mathrm{G})$ with the label $\ell_{\mathrm{G}}(e)=\left(\gamma^{-}, t, \gamma^{+}\right)$and note that since $\sigma^{\prime}$ is a homomorphism, then

$$
\sigma_{E}^{\prime}(e) \in E\left([\mathrm{C}, \mathrm{H}]_{\Gamma}^{*}\right) \quad \text { and } \quad \ell_{[\mathrm{C}, \mathrm{H}]_{\Gamma}^{*}}(e)=\left(\alpha_{i} \gamma^{-}, t, \alpha_{j} \gamma^{+}\right)
$$

Therefore, by definition of the exponential graph, we have a homomorphism

$$
\tilde{\sigma}^{e}=\left(\tilde{\sigma}_{V}^{e}, \tilde{\sigma}_{E}^{e}\right) \in \operatorname{Hom}_{\ell}\left(\mathrm{C}^{t}\left(\mathbf{y}^{t} \alpha_{i} \gamma^{-}, \mathbf{z}^{t} \alpha_{j} \gamma^{+},\left(\gamma^{-}, \gamma^{+}\right) \epsilon^{t}\right), \mathrm{H}\right),
$$

and consequently, we obtain a homomorphism

$$
\sigma^{e}=\left(\sigma_{V}^{e}, \sigma_{E}^{e}\right) \in \operatorname{Hom}_{\ell}\left(\mathrm{C}^{t}\left(\mathbf{y}^{t} \gamma^{-}, \mathbf{z}^{t} \gamma^{+},\left(\gamma^{-}, \gamma^{+}\right) \epsilon^{t}\right), \mathrm{H}\right),
$$

such that

$$
\sigma_{V}^{e}\left(\mathbf{y}^{t} \gamma^{-}\right)=\mathbf{u}^{i} \alpha_{i} \text { and } \sigma_{V}^{e}\left(\mathbf{z}^{t} \gamma^{+}\right)=\mathbf{u}^{j} \alpha_{j} .
$$

Now, by Proposition 10, these homomorphisms are compatible at each vertex and we may define a global homomorphism

$$
s_{\mathrm{G}, \mathrm{H}}\left(\left(\sigma_{V}^{\prime}, \sigma_{E}^{\prime}\right)\right)=\left(\sigma_{V}, \sigma_{E}\right) \stackrel{\text { def }}{=} \bigcup_{e \in E(\mathrm{G})} \sigma^{e} \in \operatorname{Hom}_{\ell}\left(\mathrm{G} \otimes_{\Gamma} \mathrm{C}, \mathrm{H}\right) .
$$

$(b),(c)$ :

It is enough to prove that

$$
\forall \sigma^{\prime} \in \operatorname{Hom}_{\Gamma, \mathrm{m}}\left(\mathrm{G},[\mathrm{C}, \mathrm{H}]_{\Gamma}^{*}\right) \quad r_{\mathrm{G}, \mathrm{H}}\left(s_{\mathrm{G}, \mathrm{H}}\left(\sigma^{\prime}\right)\right)=\sigma^{\prime},
$$

but by definitions,

$$
r_{\mathrm{G}, \mathrm{H}}\left(s_{\mathrm{G}, \mathrm{H}}\left(\sigma^{\prime}\right)\right)(e)=r_{\mathrm{G}, \mathrm{H}}\left(\sigma^{e}\right)(e)=\sigma^{\prime}(e),
$$

since $\left\langle\mathbf{u}^{i} \alpha_{i}\right\rangle_{\Gamma}=\left\langle\mathbf{u}^{i}\right\rangle_{\Gamma}$. Also, for any $e \in E(\mathrm{G})$ whose label is $\left(\gamma^{-}, t, \gamma^{+}\right)$, by definitions we know that

$$
r_{\mathrm{G}, \mathrm{H}}\left(s_{\mathrm{G}, \mathrm{H}}\left(\sigma_{E}^{\prime}\right)\right)(e)=e^{\prime},
$$

where

$$
\iota\left(e^{\prime}\right)=\left\langle\mathbf{u}^{i}\right\rangle_{\Gamma}=\mathbf{u}^{i} \alpha_{i}, \quad \tau\left(e^{\prime}\right)=\left\langle\mathbf{u}^{j}\right\rangle_{\Gamma}=\mathbf{u}^{j} \alpha_{j}, \quad \ell_{[\mathrm{C}, \mathrm{H}]_{\Gamma}}\left(e^{\prime}\right)=\left(\alpha_{i} \gamma^{-}, t, \alpha_{j} \gamma^{+}\right),
$$

and by Proposition 10 it is clear that

$$
r_{\mathrm{G}, \mathrm{H}}\left(s_{\mathrm{G}, \mathrm{H}}\left(\sigma_{E}^{\prime}\right)\right)(e)=\sigma_{E}^{\prime}(e)
$$

and consequently,

$$
r_{\mathrm{G}, \mathrm{H}}\left(s_{\mathrm{G}, \mathrm{H}}\left(\sigma_{E}^{\prime}\right)\right)=\sigma_{E}^{\prime} .
$$


Similarly, one may prove the following version of this theorem for symmetric constructs (we just state the main duality in this case).

Theorem 23. Let $\mathrm{C}=\left\{\mathrm{C}^{j}\left(\mathbf{y}^{j}, \mathbf{z}^{j}, \epsilon^{j}\right) \mid j \in \widehat{1 . . . m}\right\}$ be a set of $\Gamma$-coherent bsymmetric $(t, k)$-cylinders, and also, let $\mathrm{G}$ be an undirected $(\Gamma, m)$-graph. Then for any graph $\mathrm{H}$ for which $[\mathrm{C}, \mathrm{H}]_{\Gamma}^{s}$ is defined,

$$
\operatorname{Hom}_{\ell}\left(\mathrm{G} \otimes_{\Gamma}^{s} \mathrm{C}, \mathrm{H}\right) \neq \emptyset \Leftrightarrow \operatorname{Hom}_{\Gamma, \mathrm{m}}\left(\mathrm{G},[\mathrm{C}, \mathrm{H}]_{\Gamma}^{s}\right) \neq \emptyset .
$$

By the results of the next section it is clear that the proposed tensor-hom duality is actually an adjunction between the thin categories $\mathbf{L} \mathbf{G r p h} \mathbf{r}^{\leqslant}(\Gamma, m)$ and $\mathbf{L} \mathbf{G r p h} \mathbf{h}^{\leqslant}$, however the maps $r_{\mathrm{G}, \mathrm{H}}$ and $s_{\mathrm{G}, \mathrm{H}}$ are not necessarily inverse to each other, and consequently, the duality in general is not an adjunction between categories $\mathbf{L G r p h}(\Gamma, m)$ and $\mathbf{L G r p h}$ (for more on this and tight cylinders see [7]).

\subsection{Functorial properties}

Let $\mathrm{C}=\left\{\mathrm{C}^{j}\left(\mathbf{y}^{j}, \mathbf{y}^{j}, \epsilon^{j}\right) \quad \mid j \in \widehat{1 . . . m}\right\}$ be a $\Gamma$-coherent set of $(t, k)$-cylinders. We show that the cylindrical construction $-\bigotimes_{\Gamma} \mathrm{C}: \mathbf{L G r p h}(\Gamma, m) \longrightarrow \mathbf{L G r p h}$ and the exponential graph construction $[\mathrm{C},-]_{\Gamma}: \mathbf{L G r p h} \longrightarrow \mathbf{L G r p h}(\Gamma, m)$ introduce well-defined functors. To see this, for two objects $\mathrm{G}$ and $\mathrm{G}^{\prime}$ in $\operatorname{Obj}(\mathbf{L G r p h}(\Gamma, m))$, where

$$
V(\mathrm{G})=\left\{v_{1}, v_{2}, \ldots, v_{n}\right\}, \quad V\left(\mathrm{G}^{\prime}\right)=\left\{u_{1}, u_{2}, \ldots, u_{n}\right\}
$$

consider the following definition for the cylindrical construction functor,

$$
\begin{gathered}
\forall \mathrm{G} \in \operatorname{Obj}(\operatorname{LGrph}(\Gamma, m)) \quad\left(-\bigotimes_{\Gamma} \mathrm{C}\right)(\mathrm{G}) \stackrel{\text { def }}{=} \mathrm{G}_{\Gamma} \mathrm{C}, \\
\forall f \in \operatorname{Hom}_{\Gamma, \mathrm{m}}\left(\mathrm{G}, \mathrm{G}^{\prime}\right) \quad\left(-\bigotimes_{\Gamma} \mathrm{C}\right)(f) \stackrel{\text { def }}{=} f \otimes_{\Gamma} \mathrm{C} \in \operatorname{Hom}_{\ell}\left(\mathrm{G} \bigotimes_{\Gamma} \mathrm{C}, \mathrm{G}^{\prime} \bigotimes_{\Gamma} \mathrm{C}\right),
\end{gathered}
$$

defined as follows, where $\left\{\alpha_{1}, \alpha_{2}, \ldots, \alpha_{n}\right\} \subseteq \Gamma$, is provided by Proposition 10 with respect to $f$,

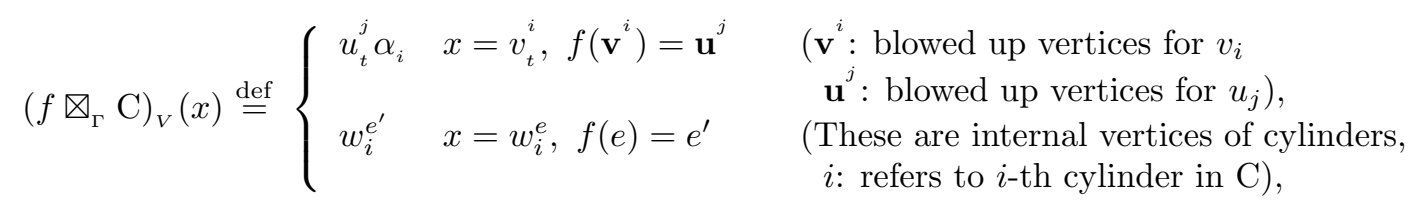

where as mentioned before $\mathbf{w}^{e} \stackrel{\text { def }}{=}\left(w_{1}^{e}, w_{2}^{e}, \ldots, w_{r}^{e}\right)$ consisting of internal vertices that appear in $\mathrm{G}_{\Gamma} \mathrm{C}$ when the edge $e$ is replaced by the corresponding cylinder (see Figure 19). 
We define

$$
\left(f \otimes_{\Gamma} \mathrm{C}\right)_{E}(e) \stackrel{\text { def }}{=}\left\{\begin{array}{l}
u_{\alpha_{i}(l)}^{j} u_{\alpha_{i}\left(l^{\prime}\right)}^{j} \\
u_{\alpha_{i}(l)}^{j} u_{\alpha_{i^{\prime}}\left(l^{\prime}\right)}^{j^{\prime}} \\
u_{\alpha_{i}(l)}^{j} w \\
w u_{\alpha_{i}(l)}^{j} \\
w w^{\prime} \in E\left(\mathrm{C}_{u_{j} u_{j^{\prime}}}^{t}\right)
\end{array}\right.
$$

$e=v_{l}^{i} v_{l^{\prime}}^{i}, f\left(v_{i}\right)=u_{j}$,

(Entire edge is in the base of a cylinder),

$e=v_{l}^{i} v_{l^{\prime}}^{i^{\prime}}, f\left(v_{i} v_{i^{\prime}}\right)=u_{j} u_{j^{\prime}}$,

(Edges which are adjacent to both bases of a cylinder),

$e=v_{l}^{i} w, f\left(v_{i}\right)=u_{j}$,

(An internal vertex and a vertex in the initial base $\mathrm{B}^{-}$),

$e=w v_{l}^{i}, f\left(v_{i}\right)=u_{j}$,

(An internal vertex and a vertex in the terminal base $\mathrm{B}^{+}$),

Strictly speaking, this describes $f \bigotimes_{\Gamma}$ - as a mapping that acts as $f$ but identically on cylinders as generalized edges.

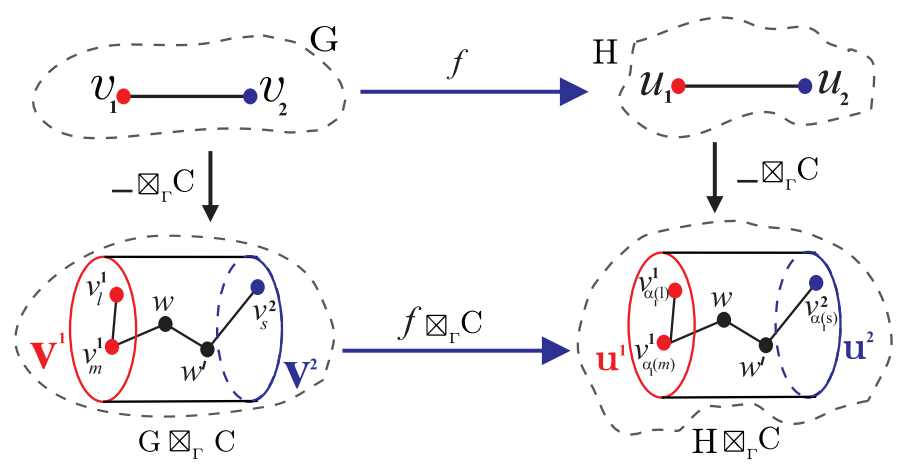

Figure 19: A commutative diagram to define $-\bigotimes_{\Gamma} \mathrm{C}(f)$.

Proposition 24. The map $-\bigotimes_{\Gamma} \mathrm{C}: \mathbf{L G r p h}(\Gamma, m) \longrightarrow \mathbf{L G r p h}$ is a well defined functor.

Proof. First, we should verify that for every $f \in \operatorname{Hom}_{\Gamma, \mathrm{m}}\left(\mathrm{G}, \mathrm{G}^{\prime}\right)$, the map $f \mathrm{\Xi}_{\Gamma} \mathrm{C}$ is actually a homomorphism in $\operatorname{Hom}_{\ell}\left(G \otimes_{\Gamma} C, G^{\prime} \bigotimes_{\Gamma} C\right)$, but this is clear since by definition not only edges but cylinders are mapped to cylinders of the same type and moreover, if $e \in E\left(\mathrm{G}_{\Gamma} \mathrm{C}\right)$, then $\left(f \nabla_{\Gamma} \mathrm{C}\right)_{E}(e)$ is an edge of $\mathrm{G}^{\prime} \bigotimes_{\Gamma} \mathrm{C}$. To see this, we consider the first two cases as follows. The rest of the cases can be verified similarly.

- $e=v_{l}^{i} v_{l^{\prime}}^{i}, f\left(v_{i}\right)=u_{j}$.

In this case we know that

$$
\left(f \bigotimes_{\Gamma} \mathrm{C}\right)_{E}(e)=u_{\alpha_{i}(l)}^{j} u_{\alpha_{i}\left(l^{\prime}\right)}^{j}
$$


where $\ell_{\mathrm{G}}^{v_{i}}(\tilde{e})=\gamma^{-}$and $\ell_{\mathrm{G}^{\prime}}^{u_{j}}(f(\tilde{e}))=\alpha_{i} \gamma^{-}$for some edge $\tilde{e} \in E(\mathrm{G})$. But if $l=\gamma^{-}\left(l_{0}\right)$ then $\alpha_{i}(l)=\alpha_{i} \gamma^{-}\left(l_{0}\right)$ which shows that both edges $e$ and $u_{\alpha_{i}(l)}^{j} u_{\alpha_{i}\left(l^{\prime}\right)}^{j}$ correspond to the same edge in the cylinder $\mathrm{C}^{\ell_{\mathrm{G}}^{*}(\tilde{e})}$.

- $e=v_{l}^{i} v_{l^{\prime}}^{i^{\prime}}, f\left(v_{i} v_{i^{\prime}}\right)=u_{j} u_{j^{\prime}}$

In this case we know that

$$
\left(f \otimes_{\Gamma} \mathrm{C}\right)_{E}(e)=u_{\alpha_{i}(l)}^{j} u_{\alpha_{i^{\prime}}\left(l^{\prime}\right)}^{j^{\prime}},
$$

where $\ell_{\mathrm{G}}\left(v_{i} v_{i^{\prime}}\right)=\left(\gamma^{-}, t, \gamma^{+}\right)$and $\ell_{\mathrm{G}}\left(u_{j} u_{j^{\prime}}\right)=\left(\alpha_{i} \gamma^{-}, t, \alpha_{i^{\prime}} \gamma^{+}\right)$. But if we assume that $l=\gamma^{-}\left(l_{0}\right)$ and $l^{\prime}=\gamma^{+}\left(l_{0}^{\prime}\right)$ then

$$
\alpha_{i}(l)=\alpha_{i} \gamma^{-}\left(l_{0}\right) \quad \text { and } \quad \alpha_{i^{\prime}}\left(l^{\prime}\right)=\alpha_{i^{\prime}} \gamma^{+}\left(l_{0}^{\prime}\right),
$$

which shows that both edges $e$ and $u_{\alpha_{i}(l)}^{j} u_{\alpha_{i^{\prime}}\left(l^{\prime}\right)}^{j^{\prime}}$ correspond to the same edge in the cylinder $\mathrm{C}^{t}$.

Also, one may easily verify that $1_{\mathrm{G}} \bigotimes_{\Gamma} \mathrm{C}=1_{\mathrm{G} \bigotimes_{\Gamma} \mathrm{C}}$ and that

$$
(f \circ g) \bigotimes_{\Gamma} \mathrm{C}=\left(f \bigotimes_{\Gamma} \mathrm{C}\right) \circ\left(g \otimes_{\Gamma} \mathrm{C}\right),
$$

for every compatible pair of homomorphisms $f$ and $g$, which shows that $-\bigotimes_{\Gamma} \mathrm{C}$ is a well-defined (covariant) functor.

On the other hand, for the exponential graph construction we have,

$$
\begin{gathered}
\forall \mathrm{H} \in \operatorname{Obj}(\mathbf{L G r p h}) \quad\left([\mathrm{C},-]_{\Gamma}\right)(\mathrm{H}) \stackrel{\text { def }}{=}[\mathrm{C}, \mathrm{H}]_{\Gamma}, \\
\forall f \in \operatorname{Hom}_{\ell}\left(\mathrm{H}, \mathrm{H}^{\prime}\right) \quad\left([\mathrm{C},-]_{\Gamma}\right)(f) \stackrel{\text { def }}{=}[\mathrm{C}, f]_{\Gamma} \in \operatorname{Hom}_{\Gamma, \mathrm{m}}\left([\mathrm{C}, \mathrm{H}]_{\Gamma},\left[\mathrm{C}, \mathrm{H}^{\prime}\right]_{\Gamma}\right),
\end{gathered}
$$

with

$$
\left([\mathrm{C}, f]_{\Gamma}\right)_{V}\left(\langle\mathbf{v}\rangle_{\Gamma}\right) \stackrel{\text { def }}{=}\langle f(\mathbf{v})\rangle_{\Gamma}=f(\mathbf{v}) \alpha_{\mathbf{v}},
$$

where $\alpha_{v}$ is given by Proposition 10 and for an edge $e$ with $e^{-}=v, e^{+}=w$ and $\ell_{[\mathrm{C}, \mathrm{H}]_{\Gamma}}(e)=$ $\left(\gamma^{-}, t, \gamma^{+}\right)$, we have

$$
\left([\mathrm{C}, f]_{\Gamma}\right)_{E}(e) \stackrel{\text { def }}{=} \tilde{e}
$$

such that

$$
\tilde{e}^{-}=\langle f(\mathbf{v})\rangle_{\Gamma}, \tilde{e}^{+}=\langle f(\mathbf{w})\rangle_{\Gamma} \text { and } \ell_{\left[\mathrm{C}, \mathrm{H}^{\prime}\right]_{\Gamma}}(\tilde{e})=\left(\alpha_{\mathbf{v}} \gamma^{-}, t, \alpha_{\mathbf{w}} \gamma^{+}\right) .
$$

Note that if $\langle f(\mathbf{v})\rangle_{\Gamma}$ is an isolated vertex in $\left[\mathrm{C}, \mathrm{H}^{\prime}\right]_{\Gamma}^{*}$, then $\langle\mathbf{v}\rangle_{\Gamma}$ is also isolated in $[\mathrm{C}, \mathrm{H}]_{\Gamma}^{*}$, and consequently, for any $\langle\mathbf{v}\rangle_{\Gamma} \in[\mathrm{C}, \mathrm{H}]_{\Gamma}$, we have $\langle f(\mathbf{v})\rangle_{\Gamma} \in\left[\mathrm{C}, \mathrm{H}^{\prime}\right]_{\Gamma}$.

Proposition 25. The map $[\mathrm{C},-]_{\Gamma}: \mathbf{L G r p h} \longrightarrow \mathbf{L G r p h}(\Gamma, m)$ is a well defined functor. 
Proof. We should verify that for every $f \in \operatorname{Hom}_{\ell}\left(\mathrm{H}, \mathrm{H}^{\prime}\right)$ the map $[\mathrm{C}, f]_{\Gamma}$ is actually a homomorphism in $\operatorname{Hom}_{\Gamma, \mathrm{m}}\left([\mathrm{C}, \mathrm{H}]_{\Gamma},\left[\mathrm{C}, \mathrm{H}^{\prime}\right]_{\Gamma}\right)$.

For this, first note that by definition of exponential graph construction and composition of homomorphisms, if $e^{-}=\mathbf{v}, e^{+}=\mathbf{w}$ and $\ell_{[\mathrm{C}, \mathrm{H}]_{\Gamma}}(e)=\left(\gamma^{-}, t, \gamma^{+}\right)$, then $\left([\mathrm{C}, f]_{\Gamma}\right)_{E}(e)$ is actually and edge of $\left[\mathrm{C}, \mathrm{H}^{\prime}\right]_{\Gamma}$ with the label $\left(\alpha_{\mathbf{v}} \gamma^{-}, t, \alpha_{\mathbf{w}} \gamma^{+}\right)$. Also, clearly by its definition and Proposition 10 this map is a homomorphism in $\operatorname{Hom}_{\Gamma, \mathrm{m}}\left([\mathrm{C}, \mathrm{H}]_{\Gamma},\left[\mathrm{C}, \mathrm{H}^{\prime}\right]_{\Gamma}\right)$.

Moreover, one may verify that $\left[\mathrm{C}, 1_{\mathrm{H}}\right]_{\Gamma}=1_{[\mathrm{C}, \mathrm{H}]_{\Gamma}}$, and that

$$
[\mathrm{C}, f \circ g]_{\Gamma}=[\mathrm{C}, f]_{\Gamma} \circ[\mathrm{C}, g]_{\Gamma}
$$

for every compatible pair of homomorphisms $f$ and $g$, which shows that $[\mathrm{C},-]_{\Gamma}$ is a well-defined (covariant) functor.

One may similarly prove that the cylindrical and the exponential constructions in the symmetric case also give rise to well-defined functors. Moreover, it is possible to prove that the section $s_{\mathrm{G}, \mathrm{H}}$ and the retraction $r_{\mathrm{G}, \mathrm{H}}$ introduced in Theorem 22 are natural with respect to their parameters $\mathrm{G}$ and $\mathrm{H}$ (see [7] for a proof).

On the other hand, although a large number of graph constructions are cylindrical, using functorial properties we prove in what follows that the classical line-graph construction for undirected graphs is not a cylindrical construct.

Proposition 26. The standard line-graph construction is not cylindrical, i.e. if $L(\mathrm{H})$ is the standard line-graph of the fixed graph $\mathrm{H}$, then there is no $\Gamma$-coherent set of cylinders $\mathrm{C}$ for which the following holds,

$$
\forall \mathrm{H}^{\prime} \approx \mathrm{H}, \quad\left[\mathrm{C}, \mathrm{H}^{\prime}\right]_{\Gamma} \approx L\left(\mathrm{H}^{\prime}\right)
$$

Proof. Assume that there exists $\mathrm{C}$ such that

$$
\forall \mathrm{H}^{\prime} \approx \mathrm{H}, \quad\left[\mathrm{C}, \mathrm{H}^{\prime}\right]_{\Gamma} \approx L\left(\mathrm{H}^{\prime}\right)
$$

Also, let $\chi(L(\mathrm{H}))=n$, and let $\mathrm{H}^{\prime} \stackrel{\text { def }}{=} \mathrm{H}(v)+\mathrm{St}_{n+1}(v)$ be the graph obtained by identifying the central vertex $v$ of the star graph on $n+1$ vertices, $\mathrm{St}_{n+1}$, with an arbitrary vertex of H. Clearly, $\mathrm{K}_{n+1} \rightarrow L\left(\mathrm{H}^{\prime}\right)$.

Note that $\mathrm{H} \approx \mathrm{H}^{\prime}$ and $L\left(\mathrm{H}^{\prime}\right) \nrightarrow L(\mathrm{H})$, i.e. $\left[\mathrm{C}, \mathrm{H}^{\prime}\right]_{\Gamma} \nrightarrow[\mathrm{C}, \mathrm{H}]_{\Gamma}$, that contradicts the functoriality of the exponential construction.

\section{Some basic applications}

This section contains some applications of the duality introduced so far. Although, these results can be of independent interest, they have been chosen to show how the basic ideas introduced in the introduction can be effectively applied. Generalizations of the proposed constructions and more detailed analysis of these methods will appear elsewhere. 


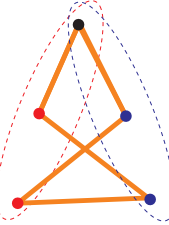

$m$

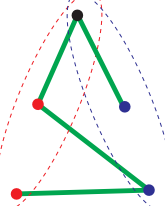

$\overrightarrow{m D}$

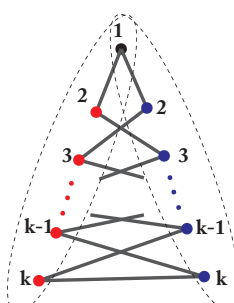

$m_{k}$

Figure 20: The Mycielski cylinder $\mathrm{mD}$, its edge perturbation $\overrightarrow{\mathrm{mD}}$ and the generalized Mycielski cylinder $\mathrm{mb}_{k}$.

\subsection{Mycielski cylinder and Generalized Grötzsch graphs}

In this section we consider the Mycielski ${ }^{7}$ cylinder and some of its variants. Although, we have already shown that cylinders whose bases have a nonempty intersection can give rise to interesting constructs (see Example 21), in this section we not only move this investigation one more step further, but we also show that the ability to use more than one cylinder in our cylindrical-exponential duality can be quite useful in some cases.

Also, it is not hard to verify that the generalized Mycielski construction of any connected graph $\mathrm{G}$ is a cylindrical construction as $\mathrm{G} \otimes \mathrm{mD}_{k}$ where $k \geqslant 3$ and $\mathrm{mb}_{k}$ is depicted in Figure 20 (see [16] and references therein for the background).

Let $\overrightarrow{\mathrm{C}}_{n}\left\langle x_{1}, x_{2}, \ldots, x_{n}\right\rangle$, where $x_{i} \in\{-,+\}$ stand for a directed cycle on $n$ vertices $\{1,2, \cdots, n\}$ where the edge $e$ is in a clockwise direction if $x_{e^{-}}=+$and is in a counterclockwise order otherwise. Also, given a (id, $m$ )-coherent set of cylinders as $\mathrm{C}$, we use a shorthand for $\overrightarrow{\mathrm{C}}_{n}\left\langle x_{1}, x_{2}, \ldots, x_{n}\right\rangle \otimes \mathrm{C}$ in which we use the name of the cylinders along with the direction specified in the $i$ th component. For instance,

$$
\overrightarrow{\mathrm{C}}_{5}\langle+\mathrm{mD},+\mathrm{mD},+\mathrm{mD},+\mathrm{mp},+\mathrm{mD}\rangle
$$

stands for the cylindrical construction on a directed five-cycle where the label of each edge corresponds to the Mycielski cylinder MD depicted in Figure 20. It is also quite easy to verify that this graph is exactly the well-known Grötzsch graph (see Figure 21(b)).

Note that our notations are consistent since whenever $x_{i} \in\{-,+\}$ we have

$$
\overrightarrow{\mathrm{C}}_{n}\left\langle x_{1}, x_{2}, \ldots, x_{n}\right\rangle=\overrightarrow{\mathrm{C}}_{n}\left\langle x_{1} \vec{I}, x_{2} \vec{I}, \ldots, x_{n} \vec{I}\right\rangle .
$$

Also, one may disregard signs when one is dealing with bsymmetric cylinders as the Mycielski cylinder $\mathrm{m}_{k}$. For instance, one may define the generalized Grötzsch graph for any $n \geqslant 3$ and $k \geqslant 3$ as

$$
\operatorname{Gr}(n, k) \stackrel{\text { def }}{=} \mathrm{C}_{n} \otimes \mathrm{m}_{k} .
$$

The following proposition reveals some coloring properties of generalized Grötzsch graphs and their edge perturbations when $k=3$ using the fundamental duality of cylindrical constructions (generalizations of these results will appear elsewhere).

\footnotetext{
[25].

${ }^{7}$ Connection to generalized Mycielski construction has been brought to our attention by Ali Taherkhani
} 


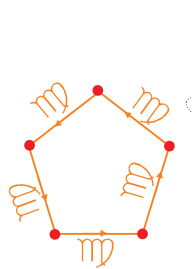

(a)

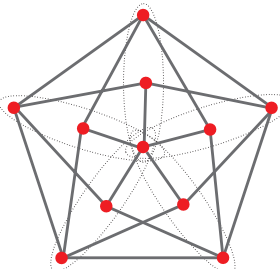

(b)

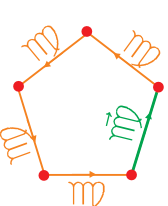

(c)

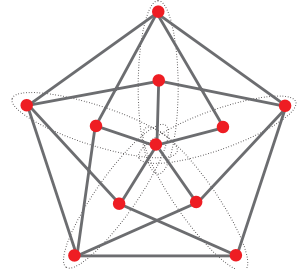

(d)

Figure 21: The Grötzsch graph and its edge perturbation.
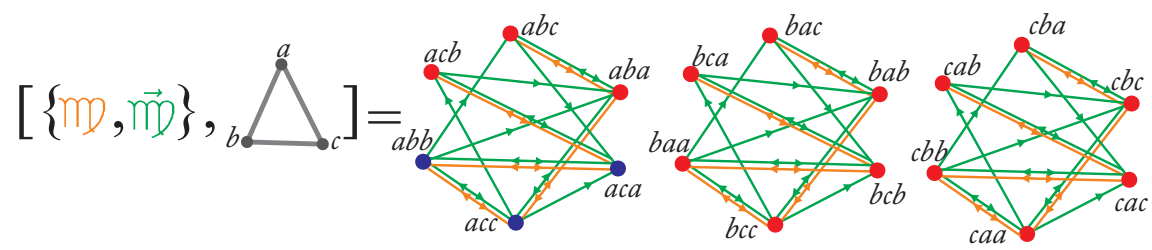

Figure 22: See Proposition 27.

Proposition 27. For $(\boldsymbol{i d}, 2)$-coherent set of cylinders $\mathrm{C} \stackrel{\text { def }}{=}\{\mathrm{mD}, \overrightarrow{\mathrm{m}}\}$ we have

(a) The cylindrical construct $\overrightarrow{\mathrm{C}}_{n}\left(x_{1}, x_{2}, \ldots, x_{n}\right)$ is 3-colorable if and only if $n$ is even or there exists $i \in \widehat{1 \ldots n}$, where $x_{i} \in\{+\overrightarrow{m p},-\overrightarrow{m p}\}$.

(b) If $n$ is odd and all $x_{i}$ 's have the same sign, then $\overrightarrow{\mathrm{C}}_{n}\left(x_{1}, x_{2}, \ldots, x_{n}\right) \not \rightarrow \mathrm{C}_{5}$. Also, when $n$ is even, for arbitrary choices of $x_{i} \in\{+\mathrm{mD},-\mathrm{mD},+\overrightarrow{\mathrm{mD}},-\overrightarrow{\mathrm{mD}}\}$ when $1 \leqslant i \leqslant n$, we have $\overrightarrow{\mathrm{C}}_{n}\left\langle x_{1}, x_{2}, \ldots, x_{n}\right\rangle \rightarrow \mathrm{C}_{5}$.

(c) For any integer $n \in \mathbb{N}$ and arbitrary choices of $x_{i} \in\{+m,-m,+\overrightarrow{m D},-\overrightarrow{m D}\}$ when $1 \leqslant i \leqslant n$, we have $\overrightarrow{\mathrm{C}}_{n+4}\left\langle-\overrightarrow{\mathrm{m}},+\overrightarrow{\mathrm{mp}},-\overrightarrow{\mathrm{mD}},+\overrightarrow{\mathrm{m}}, x_{1}, x_{2}, \ldots, x_{n}\right\rangle \rightarrow \mathrm{C}_{5}$.

(d) For any integer $n \in \mathbb{N}$ if there exists an alternating sequence of length 4 of cylinders from the set $\{+\overrightarrow{\mathrm{mD}},-\overrightarrow{\mathrm{m}}\}$ on the base graph $\overrightarrow{\mathrm{C}}_{n}$, then $\overrightarrow{\mathrm{C}}_{n}\left\langle x_{1}, x_{2}, \ldots, x_{n}\right\rangle \rightarrow \mathrm{C}_{5}$.

Proof. The exponential graphs $\left[\mathrm{C}, \mathrm{K}_{3}\right]$ is depicted in Figure 22. Also, $\left[\mathrm{C}, \mathrm{C}_{5}\right]$ consists of three isomorphic components one of which is depicted in Figure 23 to whom $\left[\mathrm{C}, \mathrm{C}_{5}\right]$ is homomorphically equivalent ${ }^{8}$.

(a) If $n$ is even then clearly there exists a homomorphism from

$$
\overrightarrow{\mathrm{C}}_{n}\left\langle\operatorname{sign}\left(x_{1}\right), \operatorname{sign}\left(x_{2}\right), \ldots, \operatorname{sign}\left(x_{n}\right)\right\rangle
$$

to the subgraph induced on the vertices $\{a b b, a c c\}$.

\footnotetext{
${ }^{8}$ Since there is no twist we have used colors to specify the cylinder corresponding to each edge for simplicity.
} 


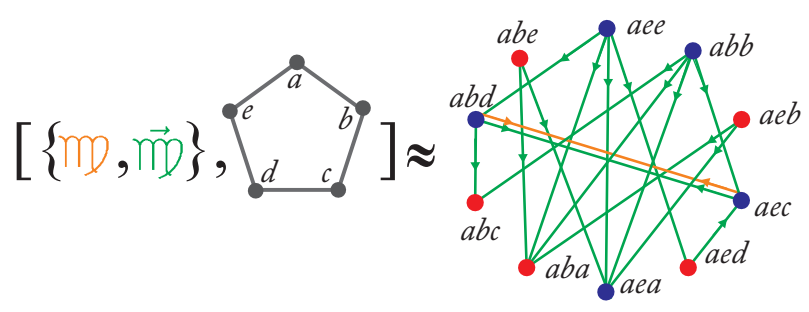

Figure 23: See Proposition 27.

On the other hand, if $n$ is odd and there exists $i \in \widehat{1 \ldots n}$, where $x_{i} \in\{+\overrightarrow{m p},-\vec{m}\}$, then there exists a homomorphism from $\overrightarrow{\mathrm{C}}_{n}\left\langle\operatorname{sign}\left(x_{1}\right), \operatorname{sign}\left(x_{2}\right), \ldots, \operatorname{sign}\left(x_{n}\right)\right\rangle$ to the subgraph induced on the vertices $\{a b b, a c c, a c a\}$.

Conversely, if $n$ is odd and for all $i \in \widehat{1 \ldots n}$ we have $x_{i} \in\{+m p,-m p\}$, since there is not any odd cycle of orange edges (corresponding to the cylinder $\mathrm{m}$ ) in $\left[\mathrm{C}_{1} \mathrm{C}_{3}\right]$, there is not any $(i d, 2)$-homomorphism from $\overrightarrow{\mathrm{C}}_{n}\left\langle x_{1}, x_{2}, \ldots, x_{n}\right\rangle$ to $\left[\mathrm{C}, \mathrm{C}_{3}\right]$.

(b) Clearly, $\left[\mathrm{C}, \mathrm{C}_{5}\right]$ does not contain any odd directed cycle, which means that there is not any $(i d, 2)$-homomorphism from $\overrightarrow{\mathrm{C}}_{n}\left\langle x_{1}, x_{2}, \ldots, x_{n}\right\rangle$ to $\left[\mathrm{C}, \mathrm{C}_{5}\right]$ if all $x_{i}{ }^{\text {'s }}$ have the same sign and $n$ is odd.

When $n$ is even there is a homomorphism to the induced subgraph on $\{a b d, a e c\}$ of $\left[\mathrm{C}, \mathrm{C}_{5}\right]$ depicted in Figure 23.

(c) If $n$ is even the statement is true by part (b). If $n$ is odd, there exists a homomorphism from $\mathrm{C}_{n}$ to the subgraph induced on $\{a b d, a e e, a e a, a b b, a e c\}$.

(d) Follows from (c) by symmetry.

\subsection{Generalized Petersen and Petersen-like graphs}

In this section we concentrate on the role of twists and we consider only the cylinder $\square$ while we show that using twists the cylindrical construction using this cylinder, which will be called $\sqcap$-lifts in the sequel, is powerful enough to give rise to some interesting results. In particular, we show that $\sqcap$-lifts can reduce the maximum degree of the base graph, and consequently, can be used to construct low degree sparse graphs of high connectivity (see Section 6 for more on this approach).

On the other hand, the results of this section can be considered as generalizations of some known results on the existence of homomorphisms from generalized Petersen graphs to the 5-cycle (e.g. see [11] for the background).

Note that, following our notations, since in this section we will be using only one cylinder in our constructions, we omit the number (or name) of the cylinder in our $(\Gamma, m)$ labels for simplicity. 
Definition 28. Let $\pi \stackrel{\text { def }}{=}\lfloor 2,1\rceil \in \mathbf{S}_{\mathbf{2}}$ where $\mathbf{S}_{\mathbf{2}} \times \mathbf{S}_{\mathbf{2}} \simeq\{(i d, i d),(i d, \pi),(\pi, i d),(\pi, \pi)\}$. The $\mathrm{S}_{2}$-graph $\mathrm{Pb}^{\ell}(n, k)$ is an undirected graph that can be constructed from the $n$-cycle $\mathrm{C}_{n}$ by connecting any pair of vertices whose distance is exactly equal to $k$, where the labeling map is

$$
\ell: E\left(\mathrm{~Pb}^{\ell}(n, k)\right) \longrightarrow \mathbf{S}_{\mathbf{2}} \times \mathbf{S}_{\mathbf{2}} .
$$

In particular, the reflexive $\mathbf{S}_{2}$-graph $\mathrm{Pb}(n, k)$ stands for the labeling when all edges of the original $n$-cycle $\mathrm{C}_{n}$ admit the label $(i d, i d)$ and the rest of edges admit the label $(\pi, \pi)$ (see Figure 24(a)). In this setting, if $\operatorname{Pet}(n, k)$ is the generalized Petersen graph (see Figure $24(b))$, then it is not hard to verify that

$$
\operatorname{Pet}(n, k)=\operatorname{Pb}(n, k) \bigotimes_{\mathbf{s}_{2}}^{s} \sqcap \text {. }
$$

Similarly, the Petersen-like graph $\operatorname{Pet}^{\ell}(n, k)$ is defined to be $\operatorname{Pb}^{\ell}(n, k) \bigotimes_{\mathbf{s}_{2}}^{s} \sqcap$.

Moreover, in what follows, $\mathrm{N} \stackrel{\text { def }}{=}\left[\sqcap, \mathrm{C}_{5}\right]_{\mathbf{S}_{2}}^{s}$ is the $\mathbf{S}_{2}$-graph of order 5 depicted in Figure 24(c) (see Example 18).

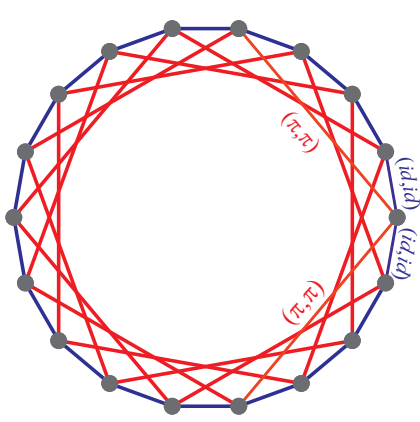

(a)

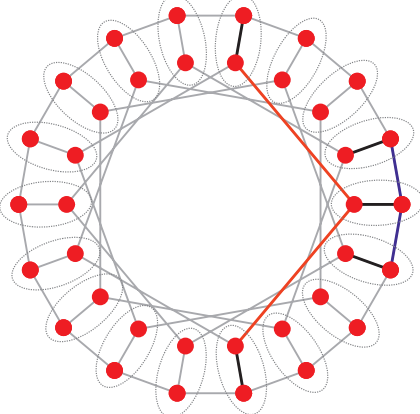

(b)

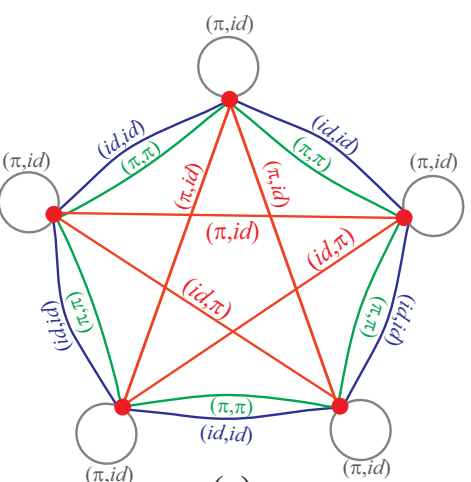

(c)

Figure 24: $(a) \operatorname{Pb}(n, k), \quad(b) \operatorname{Pet}(n, k), \quad(c) \mathrm{N} \stackrel{\text { def }}{=}\left[\sqcap, \mathrm{C}_{5}\right]_{\mathrm{S}_{2}}^{s}$.

A reflexive $\mathbf{S}_{2}$-triangle is said to be odd if both labels $(i d, i d)$ and $(\pi, \pi)$ appear on its edges. In an odd reflexively $\mathbf{S}_{2}$-labeled triangle, an odd edge is an edge whose label is different from the other two. In such a triangle, an edge whose label appears twice is said to be repeated.

Note that if a reflexively $\mathbf{S}_{2}$-labeled triangle $\mathrm{T}$ is not odd, then all labels are the same, and $\mathrm{T} \otimes \sqcap$ contains a triangle, implying that the cylindrical construct does not admit a homomorphism to the five-cycle $\mathrm{C}_{5}$. Hence, to study the $\mathrm{C}_{5}$-colorability of Petersen-like graphs we may confine ourselves to the nontrivial case when all reflexive triangles in the base graph $\mathrm{Pb}^{\ell}(n, k)$ are odd. In this situation an edge is said to be odd in $\mathrm{Pb}^{\ell}(n, k)$ if it is at least an odd edge of one reflexive triangle in this graph. The subgraph induced on all odd edges is denoted by $\mathrm{O}\left(\mathrm{Pb}^{\ell}(n, k)\right)$. Also, an extended odd subgraph in $\mathrm{Pb}^{\ell}(n, k)$ is a subgraph containing $\mathrm{O}\left(\mathrm{Pb}^{\ell}(n, k)\right)$ such that it also has at least one repeated edge from each odd reflexive triangle. 
Lemma 29. Consider the graph $\mathrm{Pb}^{\ell}(n, k)$ for an $\mathbf{S}_{2}$-labeling $\ell$. For any homomorphism $\sigma \in \operatorname{Hom}_{\mathbf{S}_{2}}\left(\mathrm{~Pb}^{\ell}(n, k), \mathrm{N}\right)$, consider $\alpha_{u}$ given by Proposition 10 as a function on the set of vertices as

$$
\alpha: V\left(\mathrm{~Pb}^{\ell}(n, k)\right) \rightarrow \mathbf{S}_{2}, \quad \alpha(u) \stackrel{\text { def }}{=} \alpha_{u} .
$$

Then,

(a) For any odd edge e we have $\alpha_{e^{+}} \neq \alpha_{e^{-}}$,

(b) If $\mathrm{O}\left(\mathrm{Pb}^{\ell}(n, k)\right)$ is a nonempty graph, then there exists an extended odd subgraph $\mathrm{H}$ such that $\mathrm{H}$ is bipartite and also $\mathrm{H}$ does not have any even cycle with alternating labels $(\pi, \pi)$ and $(i d, i d))$ of length $m$ such that $m \stackrel{5}{\equiv} 0$.

Proof.

(a) Consider a reflexive triangle in $\mathrm{Pb}^{\ell}(n, k)$, with $\gamma, \lambda \in \mathbf{S}_{2}$ and $\gamma \neq \lambda$ and also let us consider the map $\alpha$ as a vertex labeling (see Figure 25).

First, note that since $\mathrm{N}$ does not contain any reflexive triangle, $\alpha$ can not be constant on the three vertices by Lemma 14 .

Next, let the label of the odd edge be $(\gamma, \gamma)$ and by contradiction, assume that the labels at both ends of this edge be equal to $i d$ (see Figure $25(a)$ ). This implies that the label $\alpha$ should be equal to $\pi$ on the third vertex and by Proposition 10 there must be a copy of the triangle depicted in Figure $25(\mathrm{c})$ in $\mathrm{N}$ which is a contradiction.

The other case when the labels of the both ends of the odd edge is equal to $\pi$ leads to a contradiction in a similar way (see Figures $25(b)$ and $(d)$ ).

(b) By part $(a)$, we know that for any reflexive triangle in $\mathrm{Pb}^{\ell}(n, k)$, there exists exactly an odd edge and one repeated edge such that the labeling $\alpha$ assigns different labels to the ends of these edges. This shows that $\alpha$ is a 2-coloring of the subgraph $\mathrm{H}$ induced on these pairs of edges chosen from each reflexive triangle of $\mathrm{Pb}^{\ell}(n, k)$, proving that $\mathrm{H}$ is bipartite.

On the other hand, if $u_{0} u_{1} \ldots u_{2 k-1} u_{0}$ be an even cycle of length $m=2 k$ in $\mathrm{H}$ with alternating labels $\ell\left(u_{2 i} u_{2 i+1}\right)=(i d, i d)$ and $\ell\left(u_{2 i+1} u_{2 i+2}\right)=(\pi, \pi)$ for $0 \leqslant i \leqslant k-1$ with addition modulo $2 k$.

Assume that $\alpha_{u_{0}}=i d$, then since $\alpha$ is a 2-coloring by Proposition 10 we have,

$$
\ell\left(\sigma\left(u_{2 i} u_{2 i+1}\right)\right)=(i d, \pi), \quad \ell\left(\sigma\left(u_{2 i+1} u_{2 i+2}\right)\right)=(i d, \pi)
$$

again for $0 \leqslant i \leqslant k-1$ with addition modulo $2 k$.

This along with the fact that the image of any reflexively labeled triangle does not contain any loop under $\sigma$ in $\mathrm{N}$, implies that the range of $\sigma$ is the inner five-cycle of $\mathrm{N}$, and consequently, $m=2 k \stackrel{5}{\equiv} 0$.

The other case when $\alpha_{u_{0}}=\pi$ similarly gives rise to the same fact that $m=2 k \stackrel{5}{\equiv}$ 0 . 


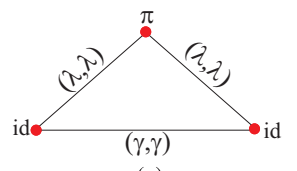

(a)

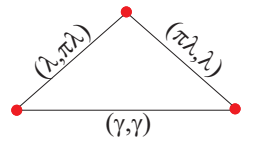

(c)

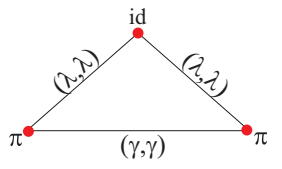

(b)

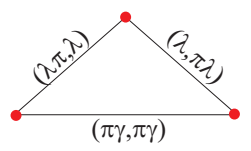

(d)

Figure 25: Reflexive triangles and their homomorphic images (see Lemma 29).

The following proposition can be considered as a generalization of a result of M. Ghebleh [11] stating that generalized Petersen graphs $\operatorname{Pet}(n, 2)$ do not admit any homomrphism to $\mathrm{C}_{5}$.

Proposition 30. For any $n \geqslant 5$, if $\mathrm{Pb}^{\ell}(n, 2)$ for an arbitrary labeling $\ell$ contains any one of the labeled subgraphs depicted in Figure 26, then $\operatorname{Pet}^{\ell}(n, 2) \nrightarrow \mathrm{C}_{5}$.

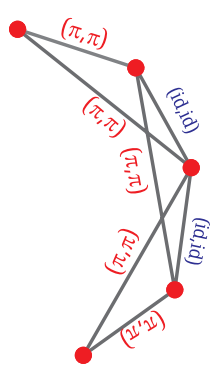

(a)

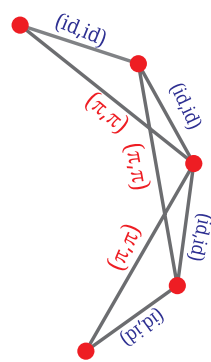

(b)

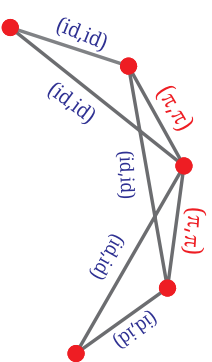

(c)

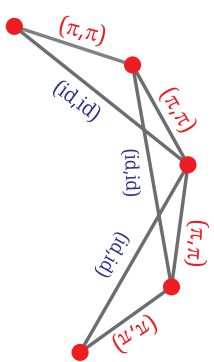

(d)

Figure 26: Obstructions for $\mathrm{C}_{5}$-colorability of $\operatorname{Pet}(n, 2)$.

Proof. Note that

$$
\begin{aligned}
\operatorname{Hom}\left(\operatorname{Pet}^{\ell}(n, k), \mathrm{C}_{5}\right) \neq \emptyset & \Leftrightarrow \operatorname{Hom}\left(\mathrm{Pb}^{\ell}(n, k) \mathbf{\Xi}_{\mathrm{S}_{2}} \sqcap \mathrm{C}_{5}\right) \neq \emptyset \\
& \Leftrightarrow \operatorname{Hom}_{\mathbf{s}_{2}}\left(\mathrm{~Pb}^{\ell}(n, k),\left[\sqcap, \mathrm{C}_{5}\right]_{\mathbf{S}_{2}}\right) \neq \emptyset \\
& \Leftrightarrow \operatorname{Hom}_{\mathbf{S}_{2}}\left(\mathrm{~Pb}^{\ell}(n, k), \mathrm{N}\right) \neq \emptyset .
\end{aligned}
$$

Hence, it suffices to verify $\operatorname{Hom}_{\mathbf{s}_{2}}\left(\mathrm{~Pb}^{\ell}(n, 2), \mathrm{N}\right)=\emptyset$.

But it is quite straight forward to verify that in cases $(a)$ and $(c)$ there exists a triangle in the induced odd subgraph, contradicting Lemma 29. Cases $(b)$ and $(d)$ follow similarly by verifying that any extended odd subgraph either contains a triangle or an alternating even cycle.

As a nonclassic example one may apply the proposition to verify that the generalized Petersen-like graph $\operatorname{Pet}^{\ell}(n, 2)$ of Figure 27 does not admit any homomorphism to $\mathrm{C}_{5}$. 
The other way round, the following result shows how the fundamental duality may be used to get a positive result.

Theorem 31. If $\frac{n}{\operatorname{gcd}(n, k)}$ is even and $\operatorname{gcd}(n, k) \geqslant 5$, then $\operatorname{Pet}(n, k) \rightarrow \mathrm{C}_{5}$.

Proof. Consider the numbering $\{0,1,2, \ldots, n-1\}$ of the vertices of $\mathrm{Pb}(n, k)$ in a clockwise order (see Figure 28(a)), and let $\operatorname{gcd}(n, k)=t$.

Let $\mathrm{Cl}_{i}$ be the cycle starting from the vertex $i \in\{0,1, \ldots, t-1\}$ on the internal edges of $\mathrm{Pb}(n, k)$ (having labels $(\pi, \pi)$ ), on the set of vertices

$$
V\left(\mathrm{Cl}_{i}\right) \stackrel{\text { def }}{=}\left\{i, i+t, i+2 t, i+3 t, \ldots, i+\left(\frac{n}{t}-1\right) t\right\}, \quad i=0,1, \ldots, t-1 .
$$

Also, for $i \in\{0,1, \ldots, t-1\}$ and $s \in\left\{0,1, \ldots,\left(\frac{n}{t}-1\right)\right\}$ we define (see Figure $28(b)$ )

$$
\alpha_{i+s t} \stackrel{\text { def }}{=} \begin{cases}\pi & s \text { is even } \\ i d & s \text { is odd }\end{cases}
$$

By hypothesis $\frac{k}{t}$ is odd, and consequently, $\alpha_{k}=\alpha_{\frac{k}{t} t}=i d$. On the other hand, since $t \geqslant 5$, there exist a reflexive closed walk $c_{0} c_{1} c_{2} \ldots c_{t-1} c_{0}$ of length $t$ on the outer cycle of $\mathrm{N}$ (having labels $(i d, i d)$ or $(\pi, \pi)$ ).

For $i \in\{0,1, \ldots, t-1\}$ and $s \in\left\{0,1, \ldots,\left(\frac{n}{t}-1\right)\right\}$, define

$$
\sigma \in \operatorname{Hom}_{\mathbf{S}_{2}}(\mathrm{~Pb}(n, k), \mathrm{N}), \quad \sigma_{V}(s t+i) \stackrel{\text { def }}{=} c_{i} .
$$

In other words we map each set $V\left(\mathrm{Cl}_{i}\right)$ to $c_{i}$ for $i=0,1,2, \ldots, t-1$.

Now, we define the mapping on the set of edges. Note that if $r t+j$ proceeds $s t+i$ on $\mathrm{Pb}(n, k)$ (with respect to the clockwise ordering of vertices), there exists an edge between $s t+i$ and $r t+j$ if

$$
\left\{\begin{array}{lll}
i=j, & & r=s+\frac{k}{t} \\
i=j+1, & 0 \leqslant j \leqslant(t-2), & r=s, \\
j=0, & i=t-1, & r=s+1 .
\end{array}\right.
$$

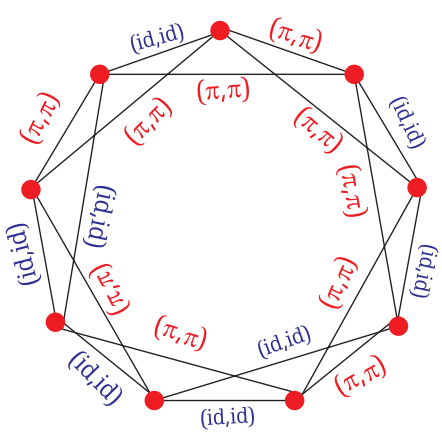

(a)

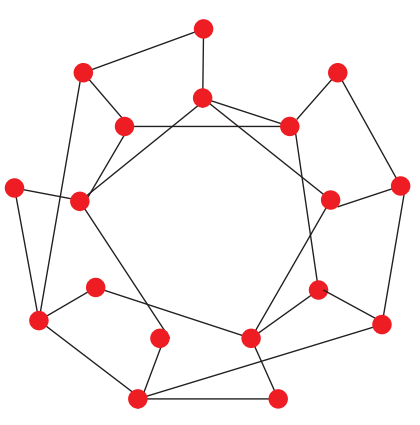

(b)

Figure 27: (a) An example for $\mathrm{Pb}^{\ell}(n, 2)$, (b) The corresponding Petersen-like graph. 


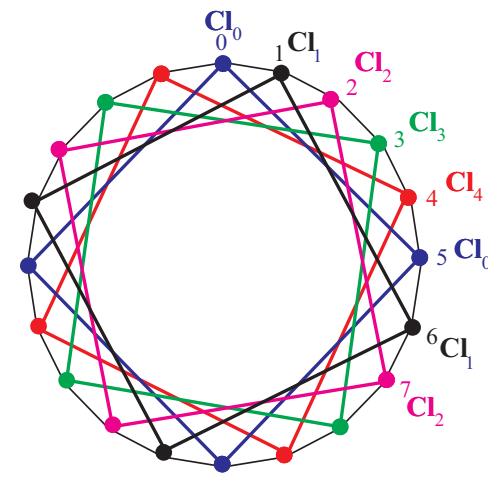

(a)

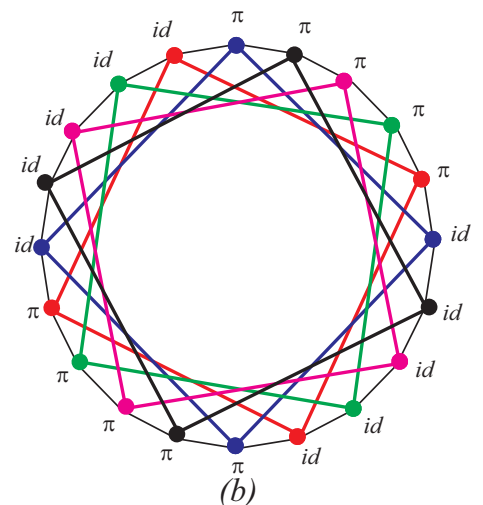

(b)

Figure 28: (a) Cycles $\mathrm{Cl}_{i}$ in $\mathrm{Pb}(20,5)$, (b) Labels $\alpha_{u}$ in $\mathrm{Pb}(20,5)$.

Hence, if $l$ is the label of the image of such an edge in $\operatorname{Pb}(n, k)$, we define,

$\sigma_{E}(s t+i, r t+j) \stackrel{\text { def }}{=}\left\{\begin{array}{lll}c_{i} c_{i} \text { (loop) } & l \in\{(\pi, i d),(i d, \pi)\} & i=j, r=s+\frac{k}{t}, \\ c_{i} c_{j} & l \in\{(i d, i d),(\pi, \pi)\} & i=j+1, j \leqslant(t-1), r=s, \\ c_{0} c_{0}(\text { loop }) & l \in\{(\pi, i d),(i d, \pi)\} & j=0, i=t-1, r=s+1 .\end{array}\right.$

Now, it is enough to show that $\sigma$ is an $\mathbf{S}_{2}$-homomorphism. Since $\frac{k}{t}$ is odd, if $i=j$ and $r=s+\frac{k}{t}$, then $\alpha_{s t+i} \neq \alpha_{r t+j}$, and consequently, label of the image of any edge is either $(\pi, i d)$ or $(i d, \pi)$. Also, if $r=s$, then $\alpha_{s t+i}=\alpha_{r t+j}$, and the label of the image of such edges, should be reflexive. Finally, if $r=s+1$, then $\alpha_{s t+i} \neq \alpha_{r t+j}$, and the image of such edge is not reflexive and must be either $(\pi, i d)$ or $(i d, \pi)$.

These observations show that

$$
\sigma \in \operatorname{Hom}_{\mathbf{s}_{2}}(\mathrm{~Pb}(n, k), \mathrm{N}),
$$

and by Theorem $22, \operatorname{Hom}\left(\operatorname{Pet}(n, k), \mathrm{C}_{5}\right) \neq \emptyset$.

Corollary 32. If $\operatorname{gcd}(s, k)=1$ and $s \geqslant 3$, then $\operatorname{Pet}(4 s k, 2 s) \rightarrow \mathrm{C}_{5}$.

\subsection{Coxeter-like graphs}

In this section our major objective is to present examples showing that even if we disregard labels in a fundamental duality we may still use it to get some interesting results. Clearly,

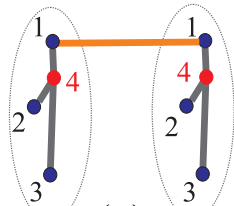

(a)

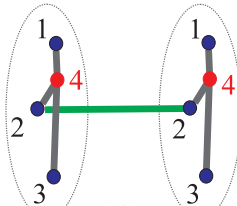

(b)

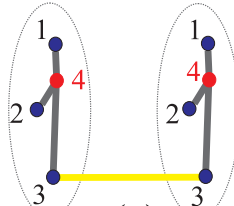

(c)

Figure 29: (a) The Coxeter cylinder $\mathfrak{C},(b) \mathfrak{C}$ after the twist $((1,2),(1,2)),(c) \mathfrak{C}$ after the twist $((1,3),(1,3))$. 


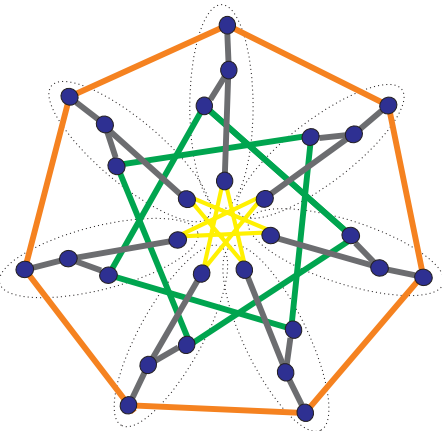

(a)

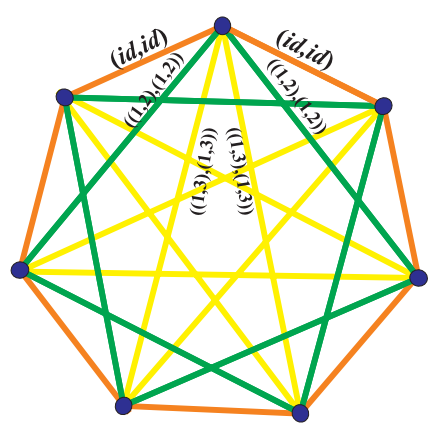

(b)

Figure 30: (a) The Coxeter graph (b) The complete $\Gamma$-graph $\mathrm{K}_{7}^{*}$ (colors represent labels).

this fact shows that the fundamental duality not only encodes a lot of information in labels (as mentioned in preceding sections) but also there is a lot of information in the structure of the exponential graphs themselves.

Let $\mathrm{K}_{7}^{*}$ be the complete graph labeled as depicted in Figures $30(b)$. It is not hard to verify that the Coxeter graph (Figures $30(a)$ ) is a cylindrical construct as

$$
\operatorname{Cox}=\mathrm{K}_{7}^{*} \otimes_{\Gamma} \mathfrak{C}
$$

where $\mathfrak{C}$ is the cylinder depicted in Figures $29(a)$ and $\Gamma \simeq \mathbf{S}_{3}$ is the subgroup of $\mathbf{S}_{4}$ fixing the element 4 in $\{1,2,3,4\}$.

Also, by the fundamental duality, we have

$$
\begin{aligned}
\operatorname{Hom}\left(\operatorname{Cox}, \mathrm{C}_{5}\right)=\emptyset & \Leftrightarrow \operatorname{Hom}\left(\mathrm{K}_{7}^{*} \bigotimes_{\Gamma} \mathfrak{C}, \mathrm{C}_{5}\right)=\emptyset \\
& \Leftrightarrow \operatorname{Hom}_{\Gamma}\left(\mathrm{K}_{7}^{*},\left[\mathfrak{C}, \mathrm{C}_{5}\right]_{\Gamma}\right)=\emptyset .
\end{aligned}
$$

In what follows we let extra brackets as $\left[\left[\mathfrak{C}, \mathrm{C}_{5}\right]\right]$ refer to the application of the forgetful functor on $\left[\mathfrak{C}, \mathrm{C}_{5}\right]_{\Gamma}$ that forgets labels in $\Gamma$-graphs.

Lemma 33. The undirected graph $\left[\left[\mathfrak{C}, \mathrm{C}_{5}\right]\right]$ is five colorable i.e.,

$$
\left[\left[\mathfrak{C}, \mathrm{C}_{5}\right]\right] \rightarrow \mathrm{K}_{5} .
$$

Proof. Fix a set of representatives for the vertex set of the graph $\left[\mathfrak{C}, \mathrm{C}_{5}\right]_{\Gamma}$ consisting of quadruples of vertices of $\mathrm{C}_{5}$. Let $\mathrm{St}_{3}$ be the star graph on four vertices. If $x_{1} x_{2} x_{3} x_{4}$ is a (by definition non-isolated) vertex in $\left[\mathfrak{C}, \mathrm{C}_{5}\right]_{\Gamma}$, there exist a homomorphism $\rho: \mathrm{St}_{3} \rightarrow$ $\mathrm{C}_{5}\left[x_{1}, x_{2}, x_{3}, x_{4}\right]$.

Let $c$ stand for the central vertex of $\mathrm{St}_{3}$ and define

$$
X_{i}=\left\{x_{1} x_{2} x_{3} x_{4} \mid \exists \rho: \mathrm{St}_{3} \rightarrow \mathrm{C}_{5}\left[x_{1}, x_{2}, x_{3}, x_{4}\right] \text { s.t. } \rho(c)=i\right\}, \quad i=0,1,2,3,4 .
$$

We claim that each $X_{i}$ is an independent set in $\left[\mathfrak{C}, \mathrm{C}_{5}\right]_{\Gamma}$. To see this, let $x_{1} x_{2} x_{3} x_{4}$ and $y_{1} y_{2} y_{3} y_{4}$ be two distinct vertices in $X_{i}$ for some $i=0,1,2,3,4$. By contradiction, if these vertices are not independent then there exists a homomorphism $\mathfrak{C} \rightarrow$ 
$\mathrm{C}_{5}\left[x_{1}, x_{2}, x_{3}, x_{4}, y_{1}, y_{2}, y_{3} y_{4}\right]$ where the left base of $\mathfrak{C}$ goes to $\mathrm{C}_{5}\left[x_{1}, x_{2}, x_{3}, x_{4}\right]$ and the right one goes to $\mathrm{C}_{5}\left[y_{1}, y_{2}, y_{3} y_{4}\right]$. Since the centers are mapped to the same vertex, this implies that there must exist a closed walk of length 3 starting and ending at this vertex in $\mathrm{C}_{5}$ which is impossible.

Let $\mathrm{K}_{7}^{\ell}$ be an arbitrary $\Gamma$-graph on $\mathrm{K}_{7}$ where $\ell$ is the $\Gamma$-labeling, then any graph construct as $\mathrm{K}_{7}^{l} \bigotimes_{\Gamma} \mathfrak{C}$ is called a Coxeter-like graph.

Theorem 34. Coxeter-like graphs are not $\mathrm{C}_{5}$-colorable i.e., for any choice for the $\Gamma$ labeling $\ell$ we have

$$
\operatorname{Hom}\left(\mathrm{K}_{7}^{l} \otimes_{\Gamma} \mathfrak{C}, \mathrm{C}_{5}\right)=\emptyset
$$

Proof. Since

$$
\operatorname{Hom}\left(\mathrm{K}_{7}^{l} \bigotimes_{\Gamma} \mathfrak{C}, \mathrm{C}_{5}\right)=\emptyset \quad \Longleftrightarrow \operatorname{Hom}_{\Gamma}\left(\mathrm{K}_{7}^{l},\left[\mathfrak{C}, \mathrm{C}_{5}\right]_{\Gamma}\right)=\emptyset
$$

it is enough to prove that $\operatorname{Hom}_{\Gamma}\left(\mathrm{K}_{7}^{l},\left[\mathfrak{C}, \mathrm{C}_{5}\right]_{\Gamma}\right)=\emptyset$.

But if $\operatorname{Hom}_{\Gamma}\left(\mathrm{K}_{7}^{l},\left[\mathfrak{C}, \mathrm{C}_{5}\right]_{\Gamma}\right) \neq \emptyset$, then applying the forgetful functor and using Lemma 33 we must have $\mathrm{K}_{7} \rightarrow\left[\left[\mathfrak{C}, \mathrm{C}_{5}\right]\right]$ which is a contradiction since $\mathrm{K}_{7}$ is not 5-colorable.

Note that even restricted to the case of Coxeter graph Theorem 34 gives a strengthening of the well-kown fact that the Coxeter graph is not a core (e.g. see $[5,12])$. The Coxeter graph as well as a couple of other Coxeter-like graphs are depicted in Figure 31.

\section{Concluding remarks}

The tensor-hom duality introduced in this article covers a large number of well-known and new graph constructions.

Firstly, it must be noted that although this duality is actually an adjunction between the thin categories of labeled and $(\Gamma, m)$-graphs, the duality is rarely an adjunction between the actual categories. Conditions under which such an adjunction holds can be of interest to establish parsimonious reductions and ought to be studied (see [15] for the general background and also [7] for the concept of tight cylinders).

Secondly, following the contents of Section 5 one should be convinced that the homomorphims problem at the level of $(\Gamma, m)$-graphs is easier to handle since a $(\Gamma, m)$-graph encodes a lot of information in its structure and its labels. Hence, an investigation for new nohomomorphism conditions at the level of $(\Gamma, m)$-graphs can be quite interesting and useful, at least to study the coloring properties of cylindrical constructs.

Thirdly, we are quite astonished to observe that a large number of extremal sparse graphs have some sort of cylindrical structure, and we believe that this observation must be studied in more detail. In particular, considering connectivity properties of cylindrical constructions can be of great importance. Results as [18] or the fact that the zig-zag product of graphs [24] is also a cylindrical construction [7] are supportive evidences in this regard and can be considered as strong motivations for such studies. In general, we believe that, considering the isoperimetric, spectral gap or other connectivity parameters 


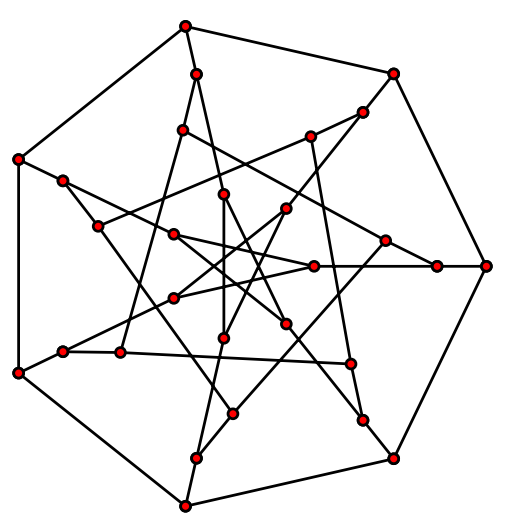

(a) The Coxeter graph,

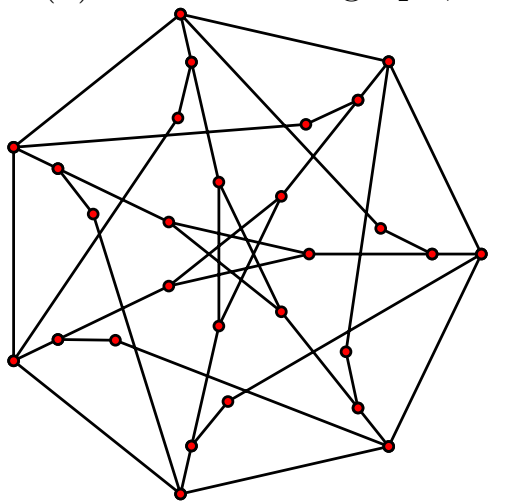

(c) A Coxeter-like graph of girth 5

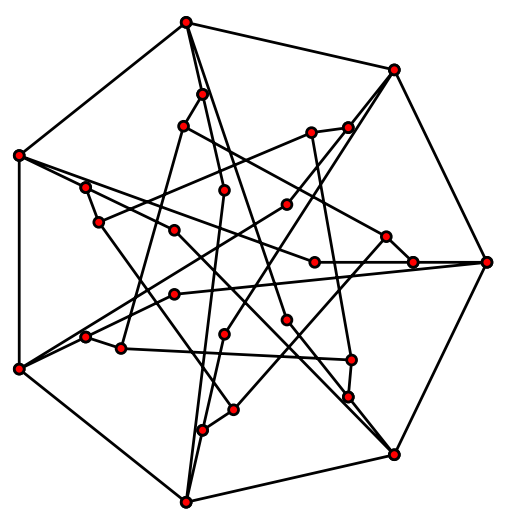

(b) A Coxeter-like graph of girth 6,

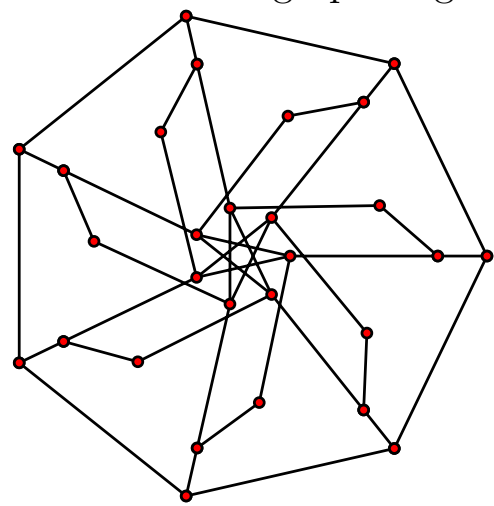

(d) A Coxeter-like graph of girth 7.

Figure 31: Some Coxeter-like graphs.

or other invariants of cylindrical constructions are of fundamental importance and are in line with the recent importance of and crucial role played by random structured matrices in mathematical sciences and physics.

Concentrating on specific cases, one may consider the iterated cylindrical constructions. It is quite interesting that one can find some related conjectures in the literature as the conjecture stating that if $n \geqslant t+2 \geqslant 3$ then the $t$ times iterated Mycielski construction of the complete graph $\mathrm{K}_{n}$ has the same chromatic and circular chromatic number [3]. On the other hand, in our opinion, the problem of finding the connectivity or chromatic properties of the $t$ times iterated $\sqcap$-lifts of the complete graph $\mathrm{K}_{2^{t}+3}$ is an interesting problem, since the procedure can lead to a 3-regular graph. Explicitly, one may ask for conditions under which there exists such a 3-regular $\sqcap$-lift that do not accept a homomorphism to the five-cycle. Also, one may ask whether the Coxeter graph is a double $\sqcap$-lift of $K_{7}$. Such questions are related to the concept of tree-based cylinders that are not discussed in this article (e.g. see [20] for more on this). 


\section{Acknowledgments}

The authors wish to thank an anonymous referee for her/his invaluable comments for improvement.

\section{References}

[1] A. Amit and N. Linial. Random graph coverings I: general theory and graph connectivity, Combinatorica, 22:1-18, 2002.

[2] O. V. Borodin and A. V. Kostochka. On an upper bound of a graph's chromatic number, depending on the graph's degree and density, Journal of Combinatorial Theory, Series B, 23:247-250, 1977.

[3] G. J. Chang, L. Huang, and X. Zhu. The circular chromatic number of Mycielskiâs graphs, Discrete Mathematics, 205:23-37, 1999.

[4] A. Daneshgar. Forcing structures and cliques in uniquely vertex colourable graphs, SIAM J. Discret. Math., 14(4):433-445, 2001.

[5] A. Daneshgar and H. Hajiabolhassan. Graph homomorphisms through random walks, J. Graph Theory, 44:15-38, 2003.

[6] A. Daneshgar, H. Hajiabolhassan, and S. Taati. On the complexity of unique list colourability and the fixing number of graphs, Ars Combinatoria, 97:301-319, 2010.

[7] A. Daneshgar, M. Hejrati, M. Madani. Cylindrical graph construction (definition and basic properties), available at arXiv:1406.3767v2, 2014.

[8] A. Daneshgar, A. J. W. Hilton and P. D. Johnson. Relations among the fractional chromatic, Hall and Hall condition numbers of simple graphs, Selected papers in honor of Helge Tverberg, Discrete Matematics, 241:189-199, 2001.

[9] A. Daneshgar, A. Rahimi, and S. Taati. Function simulation, graph grammars and colourings, International Journal of Computer Mathematics, 90(7):1334-1357, 2013.

[10] J. Foniok and C. Tardif. Adjoint functors and tree duality, Discrete Mathematics and Theoretical Computer Science, 11(2):97-110, 2009.

[11] M. Ghebleh. Theorems and computations in circular colourings of graphs, Ph.D. Thesis, Simon Fraser University, 2007.

[12] C. Godsil and G. F. Royle. Algebraic Graph Theory, Graduate Texts in Mathematics 207, Springer-Verlag, New York, 2001.

[13] A. Habel. Hyperedge Replacement: Grammars and Languages, Spriger-Verlag, Berlin Heidelberg, 1992.

[14] H. Hajiabolhassan and A. Taherkhani. Graph powers and graph homomorphisms, The Electronic Journal of Combinatorics, 17, 2010.

[15] P. Hell and J. Nešetřil. Graphs and Homomorphisms, Oxford Lecture Series in Mathematics and its Applications, Oxford University Press, Oxford, 2004. 
[16] P. C. B. Lam, G. Gu, W. Lin, and Z. Song. Circular chromatic number and a generalization of the construction of Mycielski, J. Combinatorial Theory, Series B, 89:195-205, 2003.

[17] E. R. Lamken and R. M. Wilson. Decompositions of edge-colored complete graphs, J. Combinatorial Theory, Series A, 89:149-200, 2000.

[18] A. W. Marcus, D. A. Spielman and N. Srivastava. Interlacing families I: bipartite Ramanujan graphs of all degrees, Annals of Mathematics, 182:307-325, 2015.

[19] A. Mehrabian. Personal communication, 2007.

[20] M. Madani. Graph amalgams and its applications, Ph.D. Thesis, Sharif University of Technology, (In Persian to appear), 2016.

[21] J. Nešetřil. A combinatorial classic-sparse graphs with high chromatic number, In Erdös Centennial, Bolyai Society Mathematical Society and Springer-Verlag, 25: 383407, 2013.

[22] J. Nešetřil. Aspects of structural combinatorics (graph homomorphisms and their use), Taiwanese J. Math., 3:381-424, 1999.

[23] A. Pultr. The right adjoints into the categories of relational systems, Reports of the Midwest Category Seminar, IV (Berlin), Lecture Notes in Mathematics, 117:100-113, Springer, 1970.

[24] O. Reingold, S. Vadhan and A. Wigderson. Entropy waves, the zig-zag graph product, and new constant-degree expanders, Annals of Mathematics, 155:157-187, 2002.

[25] A. Taherkhani. Personal communication, 2015. 